\title{
Repositioning of the global epicentre of non-optimal cholesterol
}

https://doi.org/10.1038/s41586-020-2338-1

Received: 18 October 2019

Accepted: 2 April 2020

Published online: 3 June 2020

Open access

Check for updates

\section{NCD Risk Factor Collaboration (NCD-RisC)*}

High blood cholesterol is typically considered a feature of wealthy western countries ${ }^{1,2}$. However, dietary and behavioural determinants of blood cholesterol are changing rapidly throughout the world ${ }^{3}$ and countries are using lipid-lowering medications at varying rates. These changes can have distinct effects on the levels of high-density lipoprotein (HDL) cholesterol and non-HDL cholesterol, which have different effects on human health ${ }^{4,5}$. However, the trends of HDL and non-HDL cholesterol levels over time have not been previously reported in a global analysis. Here we pooled 1,127 population-based studies that measured blood lipids in 102.6 million individuals aged 18 years and older to estimate trends from 1980 to 2018 in mean total, non-HDL and HDL cholesterol levels for 200 countries. Globally, there was little change in total or non-HDL cholesterol from 1980 to 2018. This was a net effect of increases in low- and middle-income countries, especially in east and southeast Asia, and decreases in high-income western countries, especially those in northwestern Europe, and in central and eastern Europe. As a result, countries with the highest level of non-HDL cholesterol-which is a marker of cardiovascular riskchanged from those in western Europe such as Belgium, Finland, Greenland, Iceland, Norway, Sweden, Switzerland and Malta in 1980 to those in Asia and the Pacific, such as Tokelau, Malaysia, The Philippines and Thailand. In 2017, high non-HDL cholesterol was responsible for an estimated 3.9 million ( $95 \%$ credible interval 3.7 million-4.2 million) worldwide deaths, half of which occurred in east, southeast and south Asia. The global repositioning of lipid-related risk, with non-optimal cholesterol shifting from a distinct feature of high-income countries in northwestern Europe, north America and Australasia to one that affects countries in east and southeast Asia and Oceania should motivate the use of population-based policies and personal interventions to improve nutrition and enhance access to treatment throughout the world.
Blood cholesterol is one of the most important risk factors for ischaemic heart disease (IHD) and ischaemic stroke ${ }^{4-6}$. Consistent and comparable information on cholesterol levels and trends in different countries can help to benchmark national performance in addressing non-optimal cholesterol, investigate the reasons behind differential trends and identify countries in which interventions are needed the most.

A previous global analysis ${ }^{7}$ reported trends in total cholesterol from 1980 to 2008, but did not analyse important lipid fractionsincluding HDL and non-HDL cholesterol-that are key to understanding the cardiovascular disease risk associated with non-optimal cholesterol. Dietary and behavioural determinants of cholesterol have changed throughout the world in the past decades, including a worldwide rise in adiposity ${ }^{8,9}$, divergent global trends in alcohol use $^{10}$, a rise in the intake of animal-source foods in middle-income countries (especially in east Asia) ${ }^{3,11}$, and a replacement of saturated fats and trans fats with unsaturated fats in some high-income countries $^{3,11,12}$. There is also considerable variation in how much different countries have adopted lipid-lowering medications ${ }^{13}$. These changes are likely to have influenced cholesterol levels substantially in the decade since the last estimates were made. Furthermore, HDL and non-HDL cholesterol, which have opposite associations with cardiovascular diseases ${ }^{4,5}$, respond differently to diet and treatment, and may therefore have different geographical patterns and trends over time $^{14}$. Information on these major lipid fractions, which were not included in the previous global estimates, is essential for priority setting and intervention choice.

Here we pooled 1,127 population-based studies that measured blood lipids in 102.6 million individuals aged 18 years and older (Extended Data Figs. 1, 2 and Supplementary Table 1) and used a Bayesian hierarchical model to estimate trends from 1980 to 2018 in mean total, non-HDL and HDL cholesterol levels for 200 countries. We also estimated the number of deaths caused by IHD and ischaemic stroke that were attributable to high levels of non-HDL cholesterol using information on its hazards from epidemiological studies. 


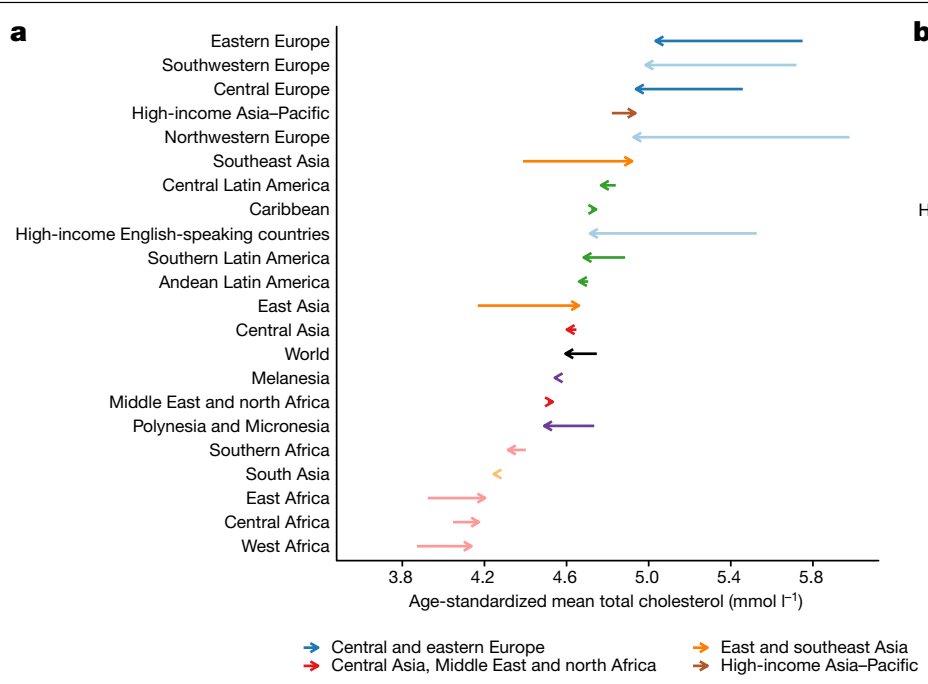

Fig. 1 Change in age-standardized mean total cholesterol between 1980 and 2018 by region for women and men. a, Age-standardized mean total cholesterol in women. b, Age-standardized mean total cholesterol in men.

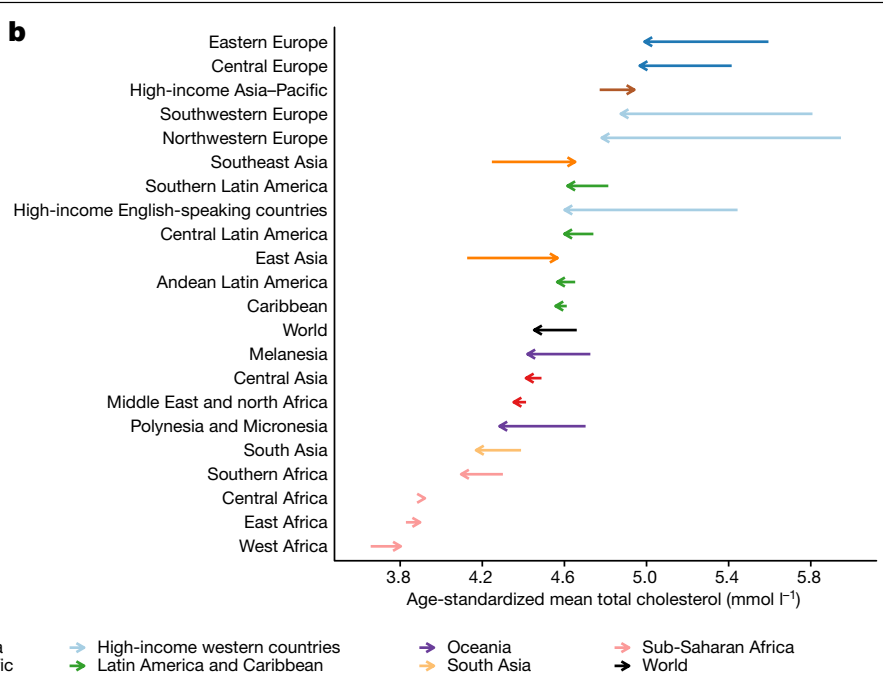

The start of the arrow shows the level in 1980 and the head indicates the level in 2018. See Extended Data Fig. 3 for age-standardized mean HDL cholesterol. One $\mathrm{mmoll}^{-1}$ is equivalent to $38.61 \mathrm{mg} \mathrm{dl}^{-1}$.

\section{Trends in total cholesterol}

In 2018, global age-standardized mean total cholesterol was $4.6 \mathrm{mmoll}^{-1}$ (95\% credible interval, 4.5-4.7) for women and $4.5 \mathrm{mmol}^{-1}(4.3-4.6)$ for men. Global age-standardized mean total cholesterol changed little over these nearly four decades, decreasing by $0.03 \mathrm{mmol}^{-1}$ per decade (-0.02-0.08) in women and $0.05 \mathrm{mmol}^{-1}$ per decade $(0.00-$ 0.11 ) in men (posterior probability of the observed declines being true declines $=0.90$ for women and 0.98 for men) (Fig. 1 ). Regionally, total cholesterol decreased the most in high-income western regions and in central and eastern Europe. The decrease was the largest (around $0.3 \mathrm{mmoll}^{-1}$ per decade; posterior probability $>0.9999$ ) in northwestern Europe, where mean total cholesterol levels had been the highest in 1980. The decrease in total cholesterol in high-income western regions and central and eastern Europe was largely due to a decline in non-HDL cholesterol (Extended Data Fig. 4), which among women was offset partly by an increase in mean HDL cholesterol levels. Mean total cholesterol changed little in most of the other regions, with the notable exception of east and southeast Asia, where it increased by more than $0.1 \mathrm{mmoll}^{-1}$ per decade in both women and men (posterior probability $\geq 0.95$ ). The increase in east and southeast Asia was largely due to an increase in non-HDL cholesterol.

\section{Trends in non-HDL and HDL cholesterol}

In 2018, global age-standardized mean non-HDL cholesterol was $3.3 \mathrm{mmol} \mathrm{l}^{-1}(3.2-3.4)$ for women and $3.3 \mathrm{mmoll}^{-1}$ (3.3-3.4) for men; global age-standardized mean HDL cholesterol was $1.3 \mathrm{mmoll}^{-1}(1.2-1.3)$ for women and $1.1 \mathrm{mmoll}^{-1}$ (1.1-1.2) for men. Global age-standardized mean non-HDL cholesterol remained almost unchanged from 1980 to 2018 , decreasing by only $0.02 \mathrm{mmoll}^{-1}$ per decade (-0.02-0.06; posterior probability $=0.80)$ in women and $0.01 \mathrm{mmoll}^{-1}$ per decade $(-0.03-$ 0.06 ; posterior probability $=0.72$ ) in men. Global age-standardized mean HDL cholesterol remained unchanged for women and decreased slightly for men (by $0.02 \mathrm{mmol} \mathrm{l}^{-1}$ per decade, posterior probability $=0.91$ ).

Regionally, non-HDL cholesterol decreased substantially in high-income western regions and central and eastern Europe. The largest decrease occurred in northwestern Europe $\left(>0.3 \mathrm{mmol} \mathrm{I}^{-1}\right.$ per decade; posterior probability $>0.9999$ ) (Fig. 2). By contrast, it increased in east and southeast Asia, parts of sub-Saharan Africa and Melanesia. The increase was the largest in southeast Asia, increasing by approximately $0.2 \mathrm{mmoll}^{-1}$ per decade (posterior probability $>0.9999$ ). Mean HDL cholesterol increased in the high-income Asia-Pacific region, by as much as $0.1 \mathrm{mmol} \mathrm{I}^{-1}$ per decade in women (posterior probability $>0.9999$ ) but decreased in Melanesia, Polynesia and Micronesia (Extended Data Fig. 3).

Belgium, Finland, Greenland, Iceland, Norway, Sweden, Switzerland and Malta had some of the highest non-HDL cholesterol levels in 1980 $\left(>4.5 \mathrm{mmol}^{-1}\right.$ in women and $>4.7 \mathrm{mmol}^{-1}$ in men) but experienced some of the largest declines (Figs. 3, 4). At the extreme, mean non-HDL cholesterol declined by around $0.45 \mathrm{mmol}^{-1}$ per decade or more in Belgian and Icelandic women and men, changing their ranks from being in the top 10 countries in terms of non-HDL cholesterol in 1980 to being ranked in the lower half of the countries in 2018-below countries in southwestern Europe such as France and Italy. The largest increases were found in east Asian countries (for example, China) and southeast Asian countries (for example, Indonesia, Thailand, Malaysia, Cambodia and Lao PDR). In these countries, age-standardized mean non-HDL cholesterol increased by as much as $0.23 \mathrm{mmoll}^{-1}$ per decade. As a result of these opposite trends, countries with the highest age-standardized mean non-HDL cholesterol levels in 2018 were all outside northwestern Europe: Tokelau, Malaysia, The Philippines and Thailand, all of which had mean non-HDL cholesterol around or above $4 \mathrm{mmoll}^{-1}$. China, which had one of the lowest mean non-HDL cholesterol levels in 1980, reached or surpassed non-HDL cholesterol levels of many high-income western countries in 2018. Sub-Saharan African countries had the lowest mean non-HDL cholesterol in 2018, as low as $2.6 \mathrm{mmol}^{-1}$ in some countries, as they had in 1980 . Not only did high-income countries benefit from decreasing non-HDL cholesterol levels, they had higher mean HDL cholesterol than low- and middle-income countries (Extended Data Fig. 6).

\section{Deaths attributable to non-optimal cholesterol}

In 2017, high non-HDL cholesterol was responsible for an estimated 3.9 million (3.7-4.2 million) worldwide deaths from IHD and ischaemic stroke (Fig. 5), accounting for a third of deaths from these causes. From 1990 to 2017, the number of deaths caused by IHD and ischaemic stroke that were attributable to high non-HDL cholesterol increased by around 910,000 globally. This increase was a net result of a large decrease in western countries, from $950,000(890,000-990,000)$ to 480,000 (430,000-530,000), and a large increase throughout Asia. In particular, the number of deaths attributable to high non-HDL cholesterol more 

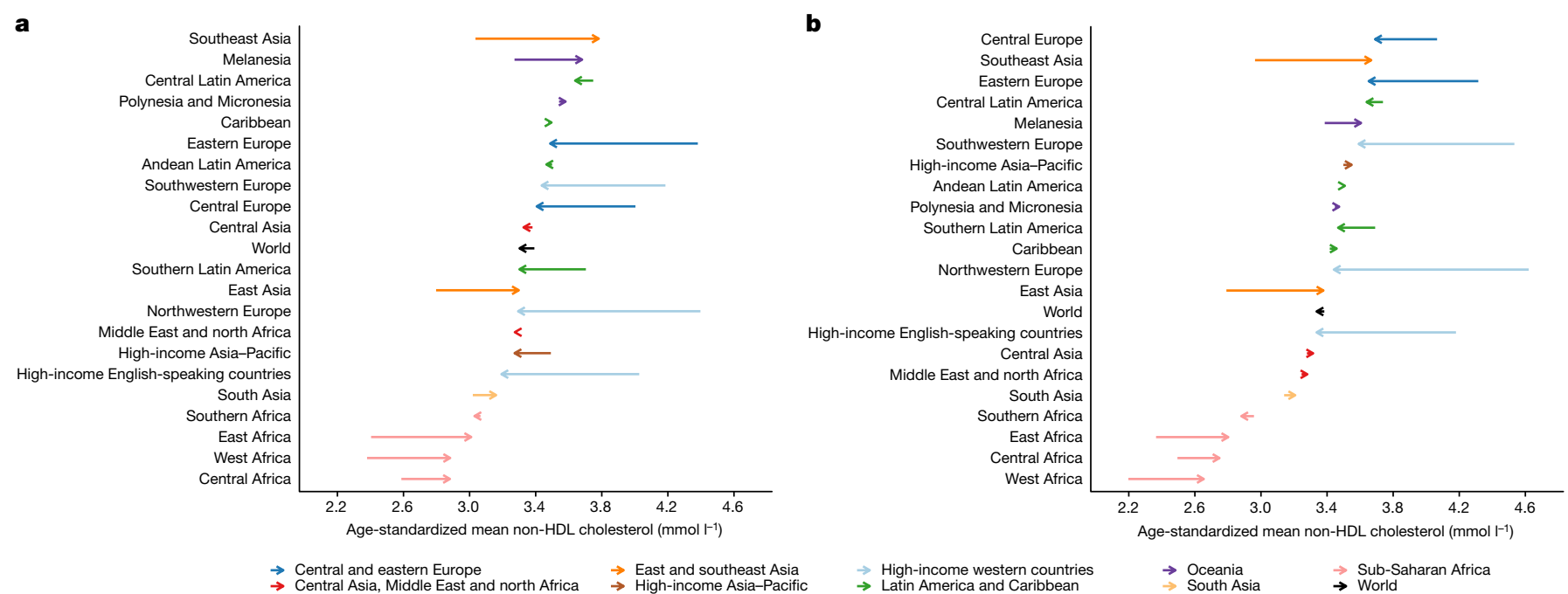

Fig. 2 | Change in age-standardized mean non-HDL cholesterol between 1980 and 2018 by region for women and men. a, Age-standardized mean non-HDL cholesterol in women. b, Age-standardized mean non-HDL

cholesterol in men. The start of the arrow shows the level in 1980 and the head indicates the level in 2018. See Extended Data Fig. 3 for age-standardized mean $\mathrm{HDL}$ cholesterol. One $\mathrm{mmoll}^{-1}$ is equivalent to $38.61 \mathrm{mg} \mathrm{dl}^{-1}$.

than tripled in east Asia, from 250,000 (230,000-270,000) to 860,000 (770,000-940,000), and more than doubled in southeast Asia, from $110,000(100,000-120,000)$ to $310,000(290,000-330,000)$. As a

a

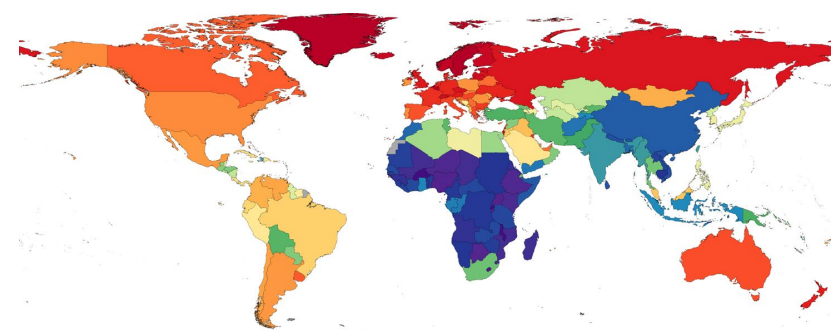

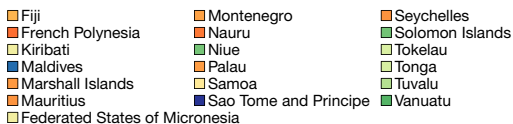

c

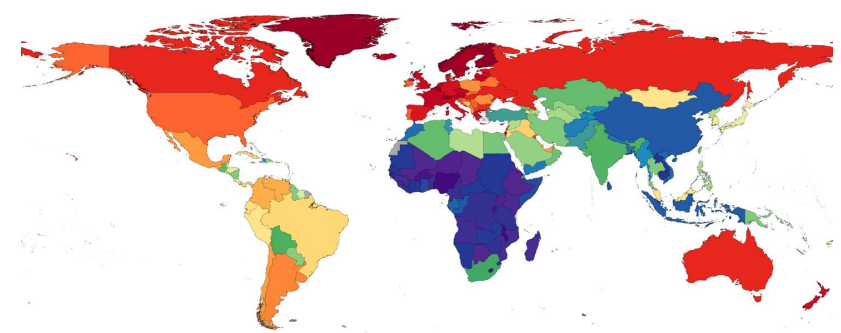

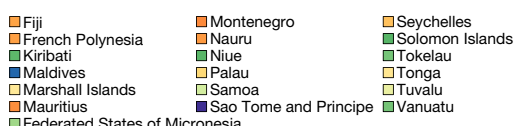

result, by 2017 east, southeast and south Asia accounted for half of all deaths attributable to high non-HDL cholesterol, compared with a quarter in 1990.

b
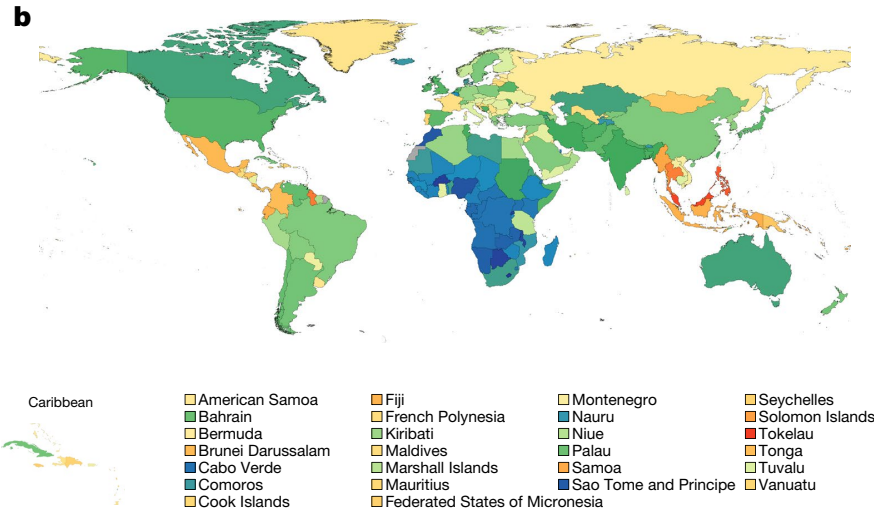

d
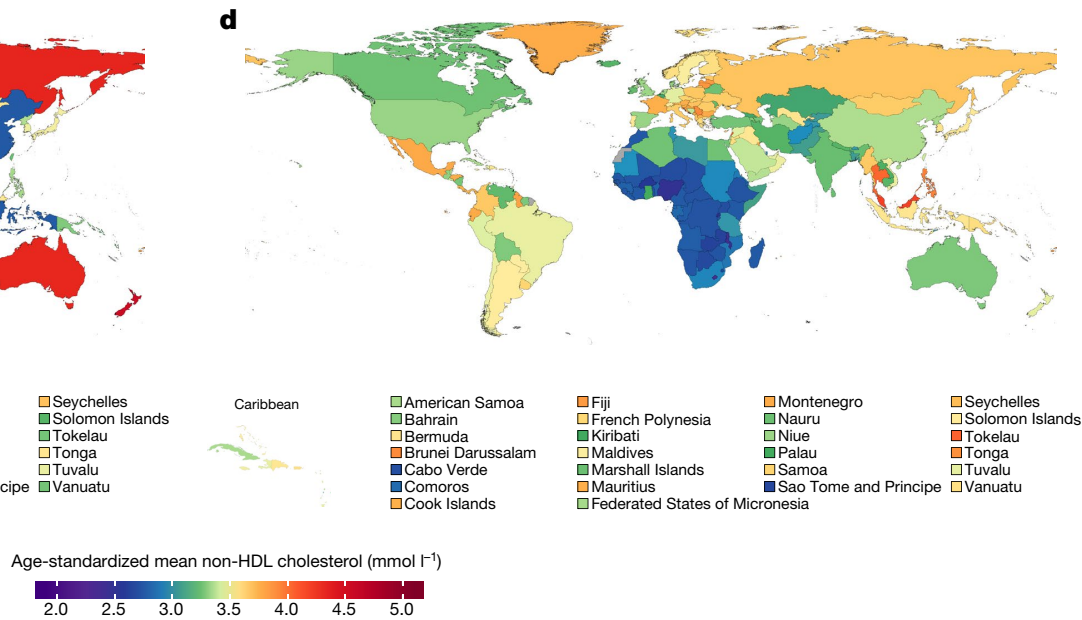

Fig. 3 | Age-standardized mean non-HDL cholesterol by country in 1980 and 2018 for women and men. a, Age-standardized mean non-HDL cholesterol in women in 1980. b. Age-standardized mean non-HDL cholesterol in women in 2018. c, Age-standardized mean non-HDL cholesterol in men in 1980. d, Age-standardized mean non-HDL cholesterol in men in 2018. See Extended Data Fig. 5 for age-standardized mean total cholesterol and Extended Data Fig. 6 for age-standardized mean HDL cholesterol. One $\mathrm{mmoll}^{-1}$ is equivalent to $38.61 \mathrm{mg} \mathrm{dl}^{-1}$. 

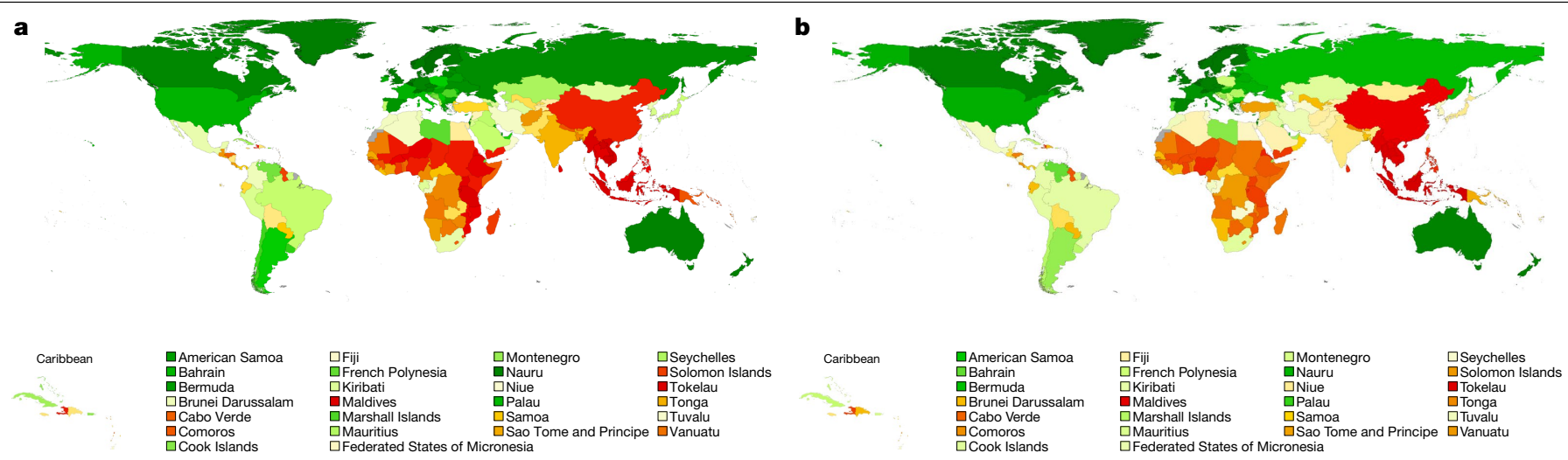

Change in age-standardized mean non-HDL cholesterol (mmol $^{-1}$ per decade)

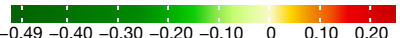

Fig. 4 | Change in age-standardized mean non-HDL cholesterol per decade by country for women and men. a, Change per decade in age-standardized mean non-HDL cholesterol in women. $\mathbf{b}$, Change per decade in age-standardized mean non-HDL cholesterol in men. See Extended Data Fig. 7 for change per decade in age-standardized mean total cholesterol and Extended Data Fig. 8 for change per decade in age-standardized mean HDL cholesterol. One $\mathrm{mmoll}^{-1}$ is equivalent to $38.61 \mathrm{mg} \mathrm{dl}^{-1}$.

\section{Implications}

Our results show that over the past nearly four decades, there has been a major global repositioning of lipid-related risk, with non-optimal cholesterol patterns shifting from being a distinct feature of high-income countries in northwestern Europe, north America and Australasia to one that affects middle-income countries in east and southeast Asia, as well as some countries in Oceania and central Latin America. This transition is especially noticeable for non-HDL cholesterol, which had not been quantified previously in a global analysis. This global repositioning has occurred as a consequence of opposing trends in high-income western countries and in Asia, which has led to some Asian countries having the highest worldwide non-HDL cholesterol levels in 2018.

The decrease in non-HDL cholesterol in western countries started in the 1980s, before statins were widely used ${ }^{15,16}$. This indicates that changes in diet, especially the replacement of saturated with unsaturated fats $\mathrm{s}^{3,17-21}$ and reduction in trans fats $\mathrm{s}^{12,17,22}$, are major contributors to this decline. Nonetheless, the increased use of statins from the late 1990s onwards ${ }^{15,16}$, may explain up to one half of the decrease in those countries in which statins are widely used ${ }^{19,23,24}$. In contrast to high-income western countries, the consumption of animal-source foods, refined carbohydrates and palm oil has increased substantially in east and southeast Asia ${ }^{3,25,26}$, where statin use remains low ${ }^{13,27}$. For example, the Pearson correlation coefficient between the change in non-HDL cholesterol and the change in a multi-dimensional score of animal-source foods and sugar ${ }^{3}$ was 0.69 for women and 0.67 for men using data from high-income western countries and countries in east and southeast Asia, the two regions that experienced the largest decrease and increase, respectively, in non-HDL cholesterol levels. Finally, changes in diet, especially a decrease in carbohydrate and an increase in fat intake ${ }^{28-31}$, may have contributed to the large increase in HDL cholesterol observed in the high-income Asia-Pacific region, where there was little increase in overweight and obesity relative to other regions $^{8,9}$. By contrast, the large increase in diabetes ${ }^{32}$ and adiposity ${ }^{8}$ in Oceania may have contributed to the decrease in HDL cholesterol in this region. The Pearson correlation coefficient between the change in HDL cholesterol and the change in body-mass index ${ }^{8}$ was -0.87 for women and -0.69 for men using countries in the high-income Asia-Pacific region and Oceania, the two regions that had the largest increase and decrease, respectively, in HDL cholesterol; the Pearson correlation coefficient for the change in HDL cholesterol and change in diabetes prevalence ${ }^{32}$ was -0.84 for women and -0.69 for men. In the same regions, the Pearson correlation coefficient between the change in non-HDL cholesterol and the change in body-mass index ${ }^{8}$ was 0.77 for women and 0.62 for men; for the change in non-HDL cholesterol and the change in diabetes prevalence ${ }^{32}$, the Pearson correlation coefficient was 0.54 for women and 0.40 for men.

Although it has previously been documented that the prevalence of adiposity $^{8,9}$, diabetes ${ }^{32}$ and high blood pressure ${ }^{33}$ is now higher in lowand middle-income countries than in high-income countries, higher cholesterol is commonly considered to be a feature of affluent western nations ${ }^{1,2}$. We show that, when focusing on non-HDL cholesterol,

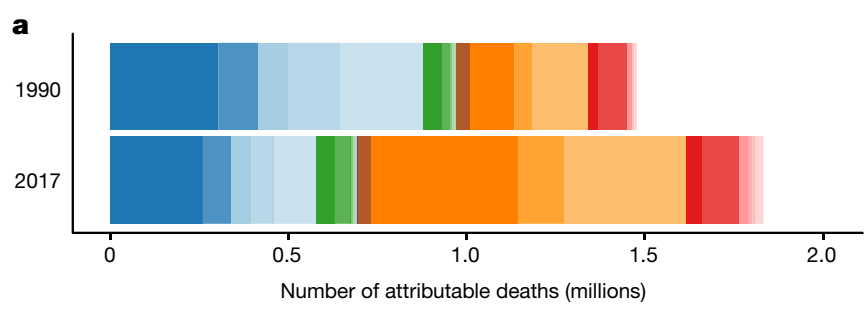

Eastern Europe
Central Europe
Southwestern Europe
Northwestern Europe
High-income English-speaking countries

Southern Latin America Central Latin America Andean Latin America Caribbean Caribbean
Polynesia and Micronesia b

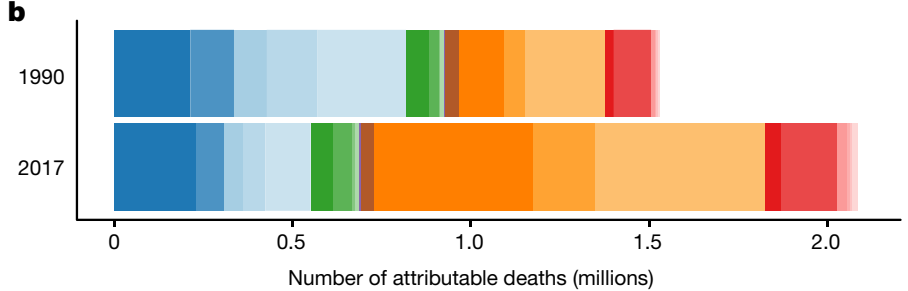

Melanesia

High-income Asia-Pacific

East Asia

Southeast Asia

South Asia

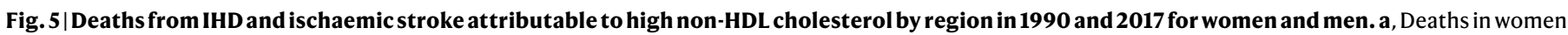
attributable to high non-HDL cholesterol. b, Deaths in men attributable to high non-HDL cholesterol. 
middle-income countries have emerged as the new global epicentre of non-optimal cholesterol as they did for other major cardiovascular disease risk factors, indicating that there is no such a thing as a western risk factor. At the same time, the populations of high-income countries would also benefit from further lowering non-HDL cholesterol. Therefore, population-based policies and personal interventions to improve nutrition and enhance treatment are now needed in all countries, especially as a part of the movement towards universal health coverage.

\section{Online content}

Any methods, additional references, Nature Research reporting summaries, source data, extended data, supplementary information, acknowledgements, peer review information; details of author contributions and competing interests; and statements of data and code availability are available at https://doi.org/10.1038/s41586-020-2338-1.

1. Danaei, G. et al. The global cardiovascular risk transition: associations of four metabolic risk factors with national income, urbanization, and Western diet in 1980 and 2008. Circulation 127, 1493-1502 (2013).

2. Ezzati, M. et al. Rethinking the "diseases of affluence" paradigm: global patterns of nutritional risks in relation to economic development. PLoS Med. 2, e133 (2005).

3. Bentham, J. et al. Multi-dimensional characterisation of global food supply from 1961 to 2013. Nat. Food 1, 70-75 (2020).

4. Prospective Studies Collaboration. Blood cholesterol and vascular mortality by age, sex and blood pressure: a meta-analysis of individual data from 61 prospective studies with 55,000 vascular deaths. Lancet 370, 1829-1839 (2007).

5. The Emerging Risk Factors Collaboration. Major lipids, apolipoproteins, and risk of vascular disease. J. Am. Med. Assoc. 302, 1993-2000 (2009).

6. Global Burden of Metabolic Risk Factors for Chronic Diseases Collaboration. Cardiovascular disease, chronic kidney disease, and diabetes mortality burden of cardiometabolic risk factors from 1980 to 2010: a comparative risk assessment. Lancet Diabetes Endocrinol. 2, 634-647 (2014).

7. Farzadfar, F. et al. National, regional, and global trends in serum total cholesterol since 1980: systematic analysis of health examination surveys and epidemiological studies with 321 country-years and 3.0 million participants. Lancet 377, 578-586 (2011).

8. NCD Risk Factor Collaboration (NCD-RisC). Worldwide trends in body-mass index underweight, overweight, and obesity from 1975 to 2016: a pooled analysis of 2416 population-based measurement studies in 128.9 million children, adolescents, and adults. Lancet 390, 2627-2642 (2017)

9. NCD Risk Factor Collaboration (NCD-RisC). Rising rural body-mass index is the main driver of the global obesity epidemic in adults. Nature 569, 260-264 (2019).

10. Manthey, J. et al. Global alcohol exposure between 1990 and 2017 and forecasts until 2030: a modelling study. Lancet 393, 2493-2502 (2019).

11. Micha, R. et al. Global, regional and national consumption of major food groups in 1990 and 2010: a systematic analysis including 266 country-specific nutrition surveys worldwide. BMJ Open 5, e008705 (2015).

12. Micha, R. et al. Global, regional, and national consumption levels of dietary fats and oils in 1990 and 2010: a systematic analysis including 266 country-specific nutrition surveys. Br. Med. J. 348, g2272 (2014)

13. Roth, G. A. et al. High total serum cholesterol, medication coverage and therapeutic control: an analysis of national health examination survey data from eight countries. Bull. World Health Organ. 89, 92-101 (2011).

14. NCD Risk Factor Collaboration (NCD-RisC). National trends in total cholesterol obscure heterogeneous changes in HDL and non-HDL cholesterol and total-to-HDL cholestero ratio: a pooled analysis of 458 population-based studies in Asian and Western countries. Int. J. Epidemiol. 49, 173-192 (2020).
15. Carroll, M. D., Kit, B. K., Lacher, D. A., Shero, S. T. \& Mussolino, M. E. Trends in lipids and lipoproteins in US adults, 1988-2010. J. Am. Med. Assoc. 308, 1545-1554 (2012).

16. Walley, T., Folino-Gallo, P., Stephens, P. \& Van Ganse, E. Trends in prescribing and utilization of statins and other lipid lowering drugs across Europe 1997-2003. Br. J. Clin. Pharmacol. 60, 543-551 (2005).

17. Vartiainen, E., Laatikainen, T., Tapanainen, H. \& Puska, P. Changes in serum cholesterol and diet in North Karelia and all Finland. Glob. Heart 11, 179-184 (2016).

18. Miller, J. C. et al. Trends in serum total cholesterol and dietary fat intakes in New Zealand between 1989 and 2009. Aust. N. Z. J. Public Health 40, 263-269 (2016).

19. Eriksson, M. et al. Greater decreases in cholesterol levels among individuals with high cardiovascular risk than among the general population: the northern Sweden MONICA study 1994 to 2014. Eur. Heart J. 37, 1985-1992 (2016).

20. Arnett, D. K. et al. Twenty-year trends in serum cholesterol, hypercholesterolemia, and cholesterol medication use: the Minnesota Heart Survey, 1980-1982 to 2000-2002. Circulation 112, 3884-3891 (2005)

21. Houterman, S., Verschuren, W. M., Oomen, C. M., Boersma-Cobbaert, C. M. \& Kromhout, D. Trends in total and high density lipoprotein cholesterol and their determinants in The Netherlands between 1993 and 1997. Int. J. Epidemiol. 30, 1063-1070 (2001).

22. Leth, T., Jensen, H. G., Mikkelsen, A. A. \& Bysted, A. The effect of the regulation on trans fatty acid content in Danish food. Atheroscler. Suppl. 7, 53-56 (2006).

23. Kypridemos, C. et al. Quantifying the contribution of statins to the decline in population mean cholesterol by socioeconomic group in England 1991 - 2012: a modelling study. PLOS ONE 10, e0123112 (2015).

24. Ford, E. S. \& Capewell, S. Trends in total and low-density lipoprotein cholesterol among U.S. adults: contributions of changes in dietary fat intake and use of cholesterol-lowering medications. PLoS ONE 8, e65228 (2013)

25. He, Y. et al. The dietary transition and its association with cardiometabolic mortality among Chinese adults, 1982-2012: a cross-sectional population-based study. Lancet Diabetes Endocrinol. 7, 540-548 (2019).

26. Wolmarans, P. Background paper on global trends in food production, intake and composition. Ann. Nutr. Metab. 55, 244-272 (2009).

27. Zhang, M. et al. Prevalence of dyslipidemia and achievement of low-density lipoprotein cholesterol targets in Chinese adults: a nationally representative survey of 163,641 adults. Int. J. Cardiol. 260, 196-203 (2018).

28. Song, Y. \& Joung, H. A traditional Korean dietary pattern and metabolic syndrome abnormalities. Nutr. Metab. Cardiovasc. Dis. 22, 456-462 (2012).

29. Yoshiike, N., Matsumura, Y., Iwaya, M., Sugiyama, M. \& Yamaguchi, M. National Nutrition Survey in Japan. J. Epidemiol. 6, S189-S200 (1996).

30. Matsumura, Y. Nutrition trends in Japan. Asia Pac. J. Clin. Nutr. 10, S40-S47 (2001).

31. Kim, S., Moon, S. \& Popkin, B. M. The nutrition transition in South Korea. Am. J. Clin. Nutr. 71, 44-53 (2000).

32. NCD Risk Factor Collaboration (NCD-RisC). Worldwide trends in diabetes since 1980: a pooled analysis of 751 population-based studies with 4.4 million participants. Lancet $\mathbf{3 8 7}$, 1513-1530 (2016).

33. NCD Risk Factor Collaboration (NCD-RisC). Worldwide trends in blood pressure from 1975 to 2015: a pooled analysis of 1479 population-based measurement studies with $19 \cdot 1$ million participants. Lancet 389, 37-55 (2017).

Publisher's note Springer Nature remains neutral with regard to jurisdictional claims in published maps and institutional affiliations.

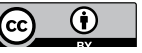

Open Access This article is licensed under a Creative Commons Attribution 4.0 International License, which permits use, sharing, adaptation, distribution and reproduction in any medium or format, as long as you give appropriate credit to the original author(s) and the source, provide a link to the Creative Commons license, and indicate if changes were made. The images or other third party material in this article are included in the article's Creative Commons license, unless indicated otherwise in a credit line to the material. If material is not included in the article's Creative Commons license and your intended use is not permitted by statutory regulation or exceeds the permitted use, you will need to obtain permission directly from the copyright holder. To view a copy of this license, visit http://creativecommons.org/licenses/by/4.0/.

(c) The Author(s), under exclusive licence to Springer Nature Limited 2020 
NCD Risk Factor Collaboration (NCD-RisC)

Cristina Taddei', Bin Zhou', Honor Bixby', Rodrigo M. Carrillo-Larco', Goodarz Danaei ${ }^{2}$, Rod T. Jackson $^{3}$, Farshad Farzadfar ${ }^{4}$, Marisa K. Sophiea', Mariachiara Di Cesare ${ }^{5}$, Maria Laura Caminia lurilli', Andrea Rodriguez Martinez', Golaleh Asghari ${ }^{6}$, Klodian Dhana ${ }^{7}$, Pablo Gulayin $^{8}$, Sujay Kakarmath ${ }^{9}$, Marilina Santero ${ }^{8}$, Trudy Voortman ${ }^{10}$, Leanne M. Riley ${ }^{11}$, Melanie J. Cowan", Stefan Savin", James E. Bennett', Gretchen A. Stevens ${ }^{1,2}$, Christopher J. Paciorek $^{13}$, Wichai Aekplakorn ${ }^{14}$, Renata Cifkova ${ }^{15,16}$, Simona Giampaoli ${ }^{17}$, Andre Pascal Kengne $^{18}$, Young-Ho Khang ${ }^{19}$, Kari Kuulasmaa ${ }^{20}$, Avula Laxmaiah ${ }^{21}$, Paula Margozzini ${ }^{22}$, Prashant Mathur ${ }^{23}$, Børge G. Nordestgaard ${ }^{24}$, Dong Zhao ${ }^{25}$, Mette Aadahl ${ }^{26}$, Leandra Abarca-Gómez ${ }^{27}$, Hanan Abdul Rahim ${ }^{28}$, Niveen M. Abu-Rmeileh ${ }^{29}$, Benjamin Acosta-Cazares ${ }^{30}$, Robert J. Adams ${ }^{31}$, Imelda A. Agdeppa ${ }^{32}$, Javad Aghazadeh-Attari ${ }^{33}$, Carlos A. Aguilar-Salinas ${ }^{34}$, Charles Agyemang ${ }^{35}$, Tarunveer S. Ahluwalia ${ }^{36}$, Noor Ani Ahmad $^{37}$, Ali Ahmadi ${ }^{38}$, Naser Ahmadi ${ }^{4}$, Soheir H. Ahmed ${ }^{39}$, Wolfgang Ahrens ${ }^{40}$, Kamel Ajlouni $^{41}$, Monira Alarouj ${ }^{42}$, Fadia AlBuhairan ${ }^{43}$, Shahla AlDhukair ${ }^{44}$, Mohamed M. Ali $^{11}$, Abdullah Alkandari ${ }^{42}$, Ala'a Alkerwi ${ }^{45}$, Eman Aly ${ }^{46}$, Deepak N. Amarapurkar ${ }^{47}$, Philippe Amouyel $^{48,49}$, Lars Bo Andersen ${ }^{50}$, Sigmund A. Anderssen ${ }^{51}$, Ranjit Mohan Anjana ${ }^{52}$, Alireza Ansari-Moghaddam ${ }^{53}$, Hajer Aounallah-Skhiri ${ }^{54}$, Joana Araújo ${ }^{55}$, Inger Ariansen ${ }^{56}$, Tahir Aris $^{37}$, Raphael E. Arku ${ }^{57}$, Nimmathota Arlappa ${ }^{21}$, Krishna K. Aryal ${ }^{58}$, Thor Aspelund ${ }^{59}$, Maria Cecília F. Assunção ${ }^{60}$, Juha Auvinen ${ }^{61,62}$, Mária Avdicová ${ }^{63}$, Ana Azevedo ${ }^{64}$, Fereidoun Azizi ${ }^{65}$ Mehrdad Azmin $^{4}$, Nagalla Balakrishna ${ }^{21}$, Mohamed Bamoshmoosh ${ }^{66}$, Maciej Banach $^{67}$, Piotr Bandosz $^{68}$, José R. Banegas ${ }^{69}$, Carlo M. Barbagallo ${ }^{70}$, Alberto Barceló ${ }^{71}$, Amina Barkat ${ }^{72}$, Iqbal Bata $^{73}$, Anwar M. Batieha ${ }^{74}$, Assembekov Batyrbek ${ }^{75}$, Louise A. Baur ${ }^{76}$, Robert Beaglehole ${ }^{3}$, Antonisamy Belavendra ${ }^{77}$, Habiba Ben Romdhane ${ }^{78}$, Mikhail Benet ${ }^{79}$, Marianne Benn ${ }^{24}$, Salim Berkinbayev $^{80}$, Antonio Bernabe-Ortiz ${ }^{81}$, Gailute Bernotiene ${ }^{82}$, Heloisa Bettiol ${ }^{83}$, Santosh K. Bhargava $^{84}$, Yufang $\mathrm{Bi}^{85}$, Asako Bienek ${ }^{86}$, Mukharram Bikbov ${ }^{87}$, Bihungum Bista ${ }^{88}$, Peter Bjerregaard $^{89}{ }^{\text {, Espen Bjertness }}{ }^{39}$, Marius B. Bjertness ${ }^{39}{ }^{\circ}$ Cecilia Björkelund $^{90}{ }^{\text {, Katia V. Bloch }}{ }^{91}$, Anneke Blokstra ${ }^{92}$, Simona Bo ${ }^{93}$, Bernhard O. Boehm ${ }^{94}$, Jose G. Boggia ${ }^{95}$, Carlos P. Boissonnet ${ }^{96}$, Marialaura Bonaccio ${ }^{97}$, Vanina Bongard ${ }^{98}$, Rossana Borchini ${ }^{99}$, Herman Borghs $^{100}$, Pascal Bovet ${ }^{101,102}$, Imperia Brajkovich ${ }^{103}$, Juergen Breckenkamp ${ }^{104}$, Hermann Brenner $^{105}$, Lizzy M. Brewster ${ }^{35}$, Graziella Bruno ${ }^{93}$, Anna Bugge ${ }^{106}$, Markus A. Busch ${ }^{107}$, Antonio Cabrera de León ${ }^{108}$, Joseph Cacciottolo ${ }^{109}$, Günay Can ${ }^{110}$, Ana Paula C. Cândido ${ }^{111}$, Mario V. Capanzana ${ }^{32}$, Eduardo Capuano ${ }^{112}$, Vincenzo Capuano ${ }^{112}$, Viviane C. Cardoso ${ }^{83}$, Joana Carvalho ${ }^{113}$, Felipe F. Casanueva ${ }^{114}$, Laura Censi ${ }^{115}$, Charalambos A. Chadjigeorgiou ${ }^{116}$, Snehalatha Chamukuttan ${ }^{17}$, Nish Chaturvedi ${ }^{118}$, Chien-Jen Chen $^{119}$, Fangfang Chen ${ }^{120}$, Shuohua Chen ${ }^{121}$, Ching-Yu Cheng ${ }^{122}$, Bahman Cheraghian ${ }^{123}$, Angela Chetrit ${ }^{124}$, Shu-Ti Chiou $^{125}$, María-Dolores Chirlaque ${ }^{126}$, Belong $\mathrm{Cho}^{127}$, Yumi Cho ${ }^{128}$, Jerzy Chudek ${ }^{129}$, Frank Claessens $^{130}$, Janine Clarke ${ }^{131}$, Els Clays ${ }^{132}$, Hans Concin ${ }^{133}$, Susana C. Confortin ${ }^{134}$, Cyrus Cooper $^{135}$, Simona Costanzo ${ }^{97}$, Dominique Cottel ${ }^{136}$, Chris Cowell $^{76}$, Ana B. Crujeiras ${ }^{137}$, Semánová Csilla ${ }^{138}$, Liufu Cui ${ }^{121}$, Felipe V. Cureau ${ }^{139}$, Graziella D’Arrigo ${ }^{140}$, Eleonora d'Orsi ${ }^{141}$, Jean Dallongeville ${ }^{136}$, Albertino Damasceno ${ }^{142}$, Rachel Dankner ${ }^{124}$, Thomas M. Dantoft ${ }^{26}$, Luc Dauchet $^{48,49}$, Kairat Davletov ${ }^{75}$, Guy De Backer ${ }^{132}$, Dirk De Bacquer ${ }^{132}$, Giovanni de Gaetano ${ }^{97}$ Stefaan De Henauw ${ }^{132}$, Paula Duarte de Oliveira ${ }^{60}$, David De Ridder ${ }^{143}$, Delphine De Smedt ${ }^{132}$ Mohan Deepa ${ }^{52}$, Alexander D. Deev ${ }^{144}$, Abbas Dehghan', Hélène Delisle ${ }^{145}$, Elaine Dennison $^{135}$, Valérie Deschamps ${ }^{146}$, Meghnath Dhimal ${ }^{88}$, Augusto F. Di Castelnuovo ${ }^{147}$, Zivka Dika $^{148}$, Shirin Djalalinia ${ }^{149}$, Annette J. Dobson ${ }^{150}$, Chiara Donfrancesco ${ }^{17}$, Silvana P. Donoso ${ }^{151}$, Angela Döring ${ }^{152}$, Maria Dorobantu ${ }^{153}$, Nico Dragano ${ }^{154}$, Wojciech Drygas ${ }^{67,155}$, Yong Du ${ }^{107}$, Charmaine A. Duante ${ }^{32}$, Rosemary B. Duda ${ }^{156}$, Vilnis Dzerve ${ }^{157}$, Elzbieta

Dziankowska-Zaborszczyk ${ }^{67}$, Ricky Eddie ${ }^{158}$, Ebrahim Eftekhar ${ }^{159}$, Robert Eggertsen ${ }^{90}$, Sareh Eghtesad $^{4}$, Gabriele Eiben ${ }^{160}$, Ulf Ekelund ${ }^{51}$, Jalila El Ati ${ }^{161}$, Denise Eldemire-Shearer ${ }^{162}$, Marie Eliasen $^{26}{ }^{\text {, Roberto Elosua }}{ }^{163}$, Rajiv T. Erasmus ${ }^{164}$, Raimund Erbel ${ }^{165}$, Cihangir Erem ${ }^{166}$, Louise Eriksen $^{89}$, Johan G. Eriksson ${ }^{167}$, Jorge Escobedo-de la Peña ${ }^{30}$, Saeid Eslami ${ }^{168}$, Ali Esmaeili ${ }^{169}$ Alun Evans ${ }^{170}$, David Faeh ${ }^{171}$, Caroline H. Fall ${ }^{135}$, Elnaz Faramarzi ${ }^{172}$, Mojtaba Farjam ${ }^{173}$, Mohammad Reza Fattahi ${ }^{174}$, Francisco J. Felix-Redondo ${ }^{175}$, Trevor S. Ferguson ${ }^{162}$, Daniel Fernández-Bergés ${ }^{176}$, Daniel Ferrante ${ }^{177}$, Marika Ferrari ${ }^{115}$, Catterina Ferreccio ${ }^{22}$, Jean Ferrieres $^{98}$, Bernhard Föger ${ }^{133}$, Leng Huat Foo ${ }^{178}$, Ann-Sofie Forslund ${ }^{179}$, Maria Forsner ${ }^{179}$, Heba M. Fouad ${ }^{46}$, Damian K. Francis ${ }^{162}$, Maria do Carmo Franco ${ }^{180}$, Oscar H. Franco ${ }^{10}$, Guillermo Frontera ${ }^{181}$, Yuki Fujita ${ }^{182}$, Matsuda Fumihiko ${ }^{183}$, Takuro Furusawa ${ }^{183}$, Zbigniew Gaciong $^{184}$, Fabio Galvano ${ }^{185}$, Jingli Gao ${ }^{121}$, Manoli Garcia-de-la-Hera ${ }^{186}$, Sarah P. Garnett ${ }^{76}$ Jean-Michel Gaspoz ${ }^{143}$, Magda Gasull ${ }^{187}$, Andrea Gazzinelli ${ }^{188}$, Johanna M. Geleijnse ${ }^{189}$, Ali Ghanbari ${ }^{4}$, Erfan Ghasemi ${ }^{4}$, Oana-Florentina Gheorghe-Fronea ${ }^{153}$, Anup Ghimire ${ }^{190}$, Francesco Gianfagna ${ }^{147,191}$, Tiffany K. Gill ${ }^{192}$, Jonathan Giovannelli4 ${ }^{48,49}$, Glen Gironella ${ }^{32}$, Aleksander Giwercman ${ }^{193}$, David Goltzman ${ }^{194}$, Helen Gonçalves ${ }^{60}$, David A. Gonzalez-Chica ${ }^{192}$, Marcela Gonzalez-Gross ${ }^{195}$, Juan P. González-Rivas ${ }^{196}$, Clicerio González-Villalpando ${ }^{197}$, María-Elena González-Villalpando ${ }^{198}$, Angel R. Gonzalez ${ }^{199}$, Frederic Gottrand ${ }^{48}$, Sidsel Graff-Iversen ${ }^{56}$, Dušan Grafnetter ${ }^{200}$, Ronald D. Gregor ${ }^{73}$, Tomasz Grodzicki $^{201}$, Anders Grøntved ${ }^{202}$, Giuseppe Grosso ${ }^{185}$, Gabriella Gruden ${ }^{93}$, Dongfeng Gu ${ }^{203}$, Pilar Guallar-Castillón ${ }^{69}$, Ong Peng Guan ${ }^{204}$, Elias F. Gudmundsson ${ }^{205}$, Vilmundur Gudnason $^{59}$, Ramiro Guerrero ${ }^{206}$, Idris Guessous ${ }^{143}$, Johanna Gunnlaugsdottir ${ }^{205}$, Rajeev Gupta $^{207}$, Laura Gutierrez ${ }^{8}$, Felix Gutzwiller ${ }^{171}$, Seongjun $\mathrm{Ha}^{208}$, Farzad Hadaegh ${ }^{209}$, Rosa Haghshenas ${ }^{4}$, Hamid Hakimi ${ }^{169}$, lan R. Hambleton ${ }^{210}$, Behrooz Hamzeh ${ }^{211}$, Sari Hantunen ${ }^{212}$, Rachakulla Hari Kumar ${ }^{21}$, Seyed Mohammad Hashemi-Shahri ${ }^{53}$, Jun Hata ${ }^{213}$, Teresa Haugsgjerd $^{214}$, Alison J. Hayes ${ }^{76}$, Jiang He ${ }^{215}$, Yuna He ${ }^{216}$, Marleen Elisabeth Hendriks ${ }^{217}$, Ana Henriques $^{55}$, Sauli Herrala ${ }^{62}$, Ramin Heshmat ${ }^{218}$, Allan G. Hill ${ }^{135}$, Sai Yin $\mathrm{Ho}^{219}$, Suzanne C. Ho $^{220}$, Michael Hobbs ${ }^{221}$, Albert Hofman ${ }^{10}$, Reza Homayounfar ${ }^{173}$, Wilma M. Hopman ${ }^{222}$, Andrea R. V. R. Horimoto ${ }^{223}$, Claudia M. Hormiga ${ }^{224}$, Bernardo L. Horta ${ }^{60}$, Leila Houti ${ }^{225}$, Christina Howitt ${ }^{210}$, Thein Thein Htay ${ }^{226}$, Aung Soe Htet ${ }^{227}$, Maung Maung Than Htike ${ }^{227}$,
José María Huerta ${ }^{228}$, Ilpo Tapani Huhtaniemi', Martijn Huisman ${ }^{229}$, Monica L. Hunsberger ${ }^{90}$, Abdullatif S. Husseini ${ }^{29}$, Inge Huybrechts ${ }^{230}$, Nahla Hwalla ${ }^{231}$, Licia lacoviello ${ }^{97,191}$, Anna G. lannone ${ }^{112}$, Mohsen M. Ibrahim ${ }^{232}$, Norazizah Ibrahim Wong ${ }^{37}$, Iris Iglesia ${ }^{233}$, Nayu Ikeda ${ }^{234}$, M. Arfan Ikram ${ }^{10}$, Violeta lotova ${ }^{235}$, Vilma E. Irazola ${ }^{8}$, Takafumi Ishida ${ }^{236}$, Muhammad Islam ${ }^{237}$, Aziz al-Safi Ismail ${ }^{178}$, Masanori Iwasaki ${ }^{238}$, Jeremy M. Jacobs ${ }^{239}$, Hashem Y. Jaddou ${ }^{74}$, Tazeen Jafar $^{122}$, Kenneth James ${ }^{162}$, Konrad Jamrozik ${ }^{192,448}$, Imre Janszky ${ }^{240}$, Edward Janus ${ }^{241}$, Marjo-Riitta Jarvelin ${ }^{1,61,62}$, Grazyna Jasienska ${ }^{201}$, Ana Jelakovic ${ }^{242}$, Bojan Jelakovic ${ }^{243}$, Garry Jennings ${ }^{244}$, Gorm B. Jensen ${ }^{24}$, Seung-lyeal Jeong ${ }^{208}$, Anjani Kumar Jha ${ }^{88}$, Chao Qiang Jiang $^{245}$, Ramon O. Jimenez ${ }^{246}$, Karl-Heinz Jöckel ${ }^{165}$, Michel Joffres ${ }^{247}$, Jari J. Jokelainen ${ }^{62}$, Jost B. Jonas ${ }^{248}$, Torben Jørgensen ${ }^{26}$, Pradeep Joshi ${ }^{249}$, Farahnaz Joukar ${ }^{250}$, Jacek Józwiak ${ }^{251}$, Anne Juolevi $^{20}$, Anthony Kafatos ${ }^{252}$, Eero O. Kajantie ${ }^{20}$, Ofra Kalter-Leibovici ${ }^{124}$, Nor Azmi Kamaruddin $^{253}$, Pia R. Kamstrup ${ }^{24}$, Khem B. Karki ${ }^{254}$, Joanne Katz ${ }^{255}$, Jussi Kauhanen ${ }^{212}$, Prabhdeep Kaur ${ }^{256}$, Maryam Kavousi ${ }^{10}$, Gyulli Kazakbaeva ${ }^{87}$, Ulrich Keil ${ }^{257}$, Sirkka Keinänen-Kiukaanniemi ${ }^{62}$, Roya Kelishadi ${ }^{258}$, Maryam Keramati ${ }^{168}$, Alina Kerimkulova ${ }^{259}$, Mathilde Kersting ${ }^{260}$, Yousef Saleh Khader ${ }^{74}$, Davood Khalili' ${ }^{6}$, Mohammad Khateeb ${ }^{41}$ Motahareh Kheradmand ${ }^{261}$, Alireza Khosravi ${ }^{262}$, Ursula Kiechl-Kohlendorfer ${ }^{263}$, Stefan Kiechl $^{263}$, Japhet Killewo ${ }^{264}$, Hyeon Chang Kim ${ }^{265}$, Jeongseon Kim ${ }^{266}$, Yeon-Yong Kim ${ }^{208}$, Jurate Klumbiene ${ }^{82}$, Michael Knoflach ${ }^{263}$, Stephanie Ko ${ }^{86}$, Hans-Peter Kohler ${ }^{267}$, Iliana V. Kohler $^{267}$, Elin Kolle ${ }^{51}$, Patrick Kolsteren ${ }^{132}$, Jürgen König ${ }^{268}$, Raija Korpelainen ${ }^{61,269}$, Paul Korrovits $^{270}$, Jelena Kos ${ }^{242}$, Seppo Koskinen ${ }^{20}$, Katsuyasu Kouda ${ }^{271}$, Sudhir Kowlessur ${ }^{272}$, Wolfgang Kratzer ${ }^{273}$, Susi Kriemler ${ }^{171}$, Peter Lund Kristensen ${ }^{202}$, Steiner Krokstad ${ }^{240}$, Daan Kromhout ${ }^{274}$, Urho M. Kujala ${ }^{275}$, Pawel Kurjata ${ }^{155}$, Catherine Kyobutungi ${ }^{276}$, Fatima Zahra Laamiri $^{277}$, Tiina Laatikainen ${ }^{20}$, Carl Lachat ${ }^{132}$, Youcef Laid ${ }^{278}$, Tai Hing Lam ${ }^{219}$,

Christina-Paulina Lambrinou ${ }^{279}$, Vera Lanska ${ }^{200}$, Georg Lappas ${ }^{280}$, Bagher Larijani ${ }^{281}$, Tint Swe Latt $^{282}$, Lars E. Laugsand ${ }^{240}$, Maria Lazo-Porras ${ }^{81}$, Jeannette Lee ${ }^{283}$, Jeonghee Lee ${ }^{266}$, Nils Lehmann ${ }^{165}$, Terho Lehtimäki ${ }^{284,285}$, Naomi S. Levitt ${ }^{286}$, Yanping Li' ${ }^{2}$, Christa L. Lilly ${ }^{287}$, Wei-Yen $\operatorname{Lim}^{283}$, M. Fernanda Lima-Costa ${ }^{288}$, Hsien-Ho Lin ${ }^{289}$, Xu Lin ${ }^{290}$, Yi-Ting Lin ${ }^{291}$, Lars Lind ${ }^{291}$, Allan Linneberg $^{26}$, Lauren Lissner ${ }^{90}$, Jing Liu ${ }^{25}$, Helle-Mai Loit ${ }^{292}$, Esther Lopez-Garcia ${ }^{69}$, Tania Lopez $^{293}$, Paulo A. Lotufo ${ }^{83}$, José Eugenio Lozano ${ }^{294}$, Dalia Luksiene ${ }^{82}$, Annamari Lundqvist ${ }^{20}$, Robert Lundqvist ${ }^{295}$, Nuno Lunet ${ }^{113}$, Guansheng Ma ${ }^{296}$, George L. L. Machado-Coelho ${ }^{297}$, Aristides M. Machado-Rodrigues ${ }^{298}$, Suka Machi ${ }^{299}$, Ahmed A. Madar ${ }^{39}$, Stefania Maggi ${ }^{300}$, Dianna J. Magliano ${ }^{301}$, Emmanuella Magriplis ${ }^{302}$, Gowri Mahasampath ${ }^{77}$, Bernard Maire ${ }^{303}$, Marcia Makdisse ${ }^{304}$, Fatemeh Malekzadeh ${ }^{174}$, Reza Malekzadeh ${ }^{4}$, Kodavanti Mallikharjuna Rao $^{21}$, Yannis Manios ${ }^{279}$, Jim I. Mann ${ }^{305}$, Fariborz Mansour-Ghanaei ${ }^{250}$, Enzo Manzato ${ }^{306}$, Pedro Marques-Vidal ${ }^{307}$, Reynaldo Martorell ${ }^{308}$, Luis P. Mascarenhas ${ }^{309}$, Ellisiv B. Mathiesen $^{310}$, Tandi E. Matsha ${ }^{311}$, Christina Mavrogianni ${ }^{279}$, Shelly R. McFarlane ${ }^{162}$, Stephen T. McGarvey ${ }^{312}$, Stela McLachlan ${ }^{313}$, Rachael M. McLean ${ }^{305}$, Scott B. McLean ${ }^{131}$, Breige A. McNulty $^{314}$, Sounnia Mediene-Benchekor ${ }^{225}$, Parinaz Mehdipour ${ }^{4}$, Kirsten Mehlig ${ }^{90}$, Amir Houshang Mehrparvar ${ }^{315}$, Aline Meirhaeghe ${ }^{316}$, Christa Meisinger ${ }^{152}$, Ana Maria B. Menezes ${ }^{60}$, Geetha R. Menon ${ }^{317}$, Shahin Merat ${ }^{4}$, Alibek Mereke ${ }^{75}$, Indrapal I. Meshram ${ }^{21}$, Patricia Metcalf ${ }^{3}$, Haakon E. Meyer ${ }^{39}$, Jie Mi ${ }^{120}$, Nathalie Michels ${ }^{132}$, Jody C. Miller ${ }^{305}$, Cláudia S. Minderico ${ }^{318}$, G. K. Mini ${ }^{319}$, Juan Francisco Miquel ${ }^{22}$, J. Jaime Miranda ${ }^{81}$, Mohammad Reza Mirjaliti ${ }^{315}$, Erkin Mirrakhimov $^{259}$, Pietro A. Modesti ${ }^{320}$, Sahar Saeedi Moghaddam ${ }^{4}$, Bahram Mohajer ${ }^{4}$, Mostafa K. Mohamed ${ }^{321}$, Kazem Mohammad ${ }^{4}$, Zahra Mohammadi ${ }^{4}$, Noushin Mohammadifard ${ }^{322}$, Reza Mohammadpourhodki ${ }^{168}$, Viswanathan Mohan ${ }^{52}$, Salim Mohanna ${ }^{81}$, Muhammad Fadhli Mohd Yusoff $^{37}$, Iraj Mohebbi ${ }^{33}$, Farnam Mohebi ${ }^{4}$, Marie Moitry ${ }^{323,324}$, Line T. Møllehave ${ }^{26}$, Niels C. Møller ${ }^{202}$, Dénes Molnár ${ }^{325}$, Amirabbas Momenan ${ }^{6}$, Charles K. Mondo ${ }^{326}$, Eric Monterrubio-Flores ${ }^{197}$, Mahmood Moosazadeh ${ }^{261}$, Alain Morejon ${ }^{327}$, Luis A. Moreno ${ }^{233}$, Karen Morgan $^{328}$, Suzanne N. Morin ${ }^{194}$, George Moschonis ${ }^{329}$, Malgorzata Mossakowska ${ }^{330}$, Aya Mostafa $^{321}$, Jorge Mota ${ }^{113}$, Mohammad Esmaeel Motlagh ${ }^{123}$, Jorge Motta ${ }^{331}$, Kelias P. Msyamboza $^{332}$, Maria L. Muiesan ${ }^{333}$, Martina Müller-Nurasyid ${ }^{152}$, Jaakko Mursu ${ }^{212}$, Norlaila Mustafa $^{253}$, Iraj Nabipour ${ }^{334}$, Shohreh Naderimagham ${ }^{4}$, Gabriele Nagel ${ }^{335}$, Balkish M. Naidu ${ }^{37}$, Farid Najafi' ${ }^{211}$, Harunobu Nakamura ${ }^{336}$, Jana Námešná ${ }^{63}$, Ei Ei K. Nang ${ }^{283}$, Vinay B. Nangia ${ }^{337}$, Matthias Nauck ${ }^{338}$, William A. Neal ${ }^{287}$, Azim Nejatizadeh ${ }^{159}$, Ilona Nenko ${ }^{201}$, Flavio Nervi ${ }^{22}$, Nguyen D. Nguyen ${ }^{339}$, Quang Ngoc Nguyen ${ }^{340}$, Ramfis E. Nieto-Martínez ${ }^{341}$, Thomas Nihal ${ }^{77}$, Teemu J. Niiranen ${ }^{20,342}$, Guang Ning ${ }^{85}$, Toshiharu Ninomiya ${ }^{213}$, Marianna Noale ${ }^{300}$, Oscar A. Noboa $^{95}$, Davide Noto ${ }^{70}$, Mohannad Al Nsour ${ }^{343}$, Irfan Nuhoğlu ${ }^{166}$, Terence W. O'Neill ${ }^{344}$, Dermot O'Reilly ${ }^{170}$, Angélica M. Ochoa-Avilés ${ }^{151}$, Kyungwon Oh $^{128}$, Ryutaro Ohtsuka ${ }^{345}$, Örn Olafsson $^{205}$, Valérie Olié ${ }^{146}$, Isabel O. Oliveira ${ }^{60}$, Mohd Azahadi Omar ${ }^{37}$, Altan Onat ${ }^{346,448}$, Sok King Ong ${ }^{347}$, Pedro Ordunez ${ }^{71}$, Rui Ornelas ${ }^{348}$, Pedro J. Ortiz ${ }^{81}$, Clive Osmond ${ }^{349}$, Sergej M. Ostojic $^{350}$, Afshin Ostovar ${ }^{4}$, Johanna A. Otero ${ }^{224}$, Ellis Owusu-Dabo ${ }^{351}$, Fred Michel Paccaud $^{352}$, Elena Pahomova ${ }^{157}$, Andrzej Pajak ${ }^{201}$, Luigi Palmieri" ${ }^{17}$, Wen-Harn Pan ${ }^{119}$, Songhomitra Panda-Jonas ${ }^{248}$, Francesco Panza ${ }^{353}$, Winsome R. Parnell ${ }^{305}$, Nikhil D. Patel ${ }^{354}$, Nasheeta Peer ${ }^{355}$, Sergio Viana Peixoto ${ }^{288}$, Markku Peltonen ${ }^{20}$, Alexandre C. Pereira ${ }^{223}$, Annette Peters ${ }^{152}$, Astrid Petersmann ${ }^{338}$, Janina Petkeviciene ${ }^{82}$, Niloofar Peykari ${ }^{149}$, Son Thai Pham $^{356}$, Rafael N. Pichardo ${ }^{357}$, Iris Pigeot ${ }^{358}$, Aida Pilav ${ }^{359}$, Lorenza Pilotto ${ }^{360}$, Aleksandra Piwonska ${ }^{155}$, Andreia N. Pizarro ${ }^{113}$, Pedro Plans-Rubió ${ }^{361}$, Silvia Plata ${ }^{362}$, Hermann Pohlabeln $^{358}$, Miquel Porta ${ }^{163}$, Marileen L. P. Portegies ${ }^{10}$, Anil Poudyal ${ }^{88}$, Farhad Pourfarzi ${ }^{363}$, Hossein Poustchi ${ }^{4}$, Rajendra Pradeepa ${ }^{52}$, Jacqueline F. Price ${ }^{313}$, Rui Providencia ${ }^{118}$, Jardena J. Puder $^{307}$, Soile E. Puhakka ${ }^{61,269}$, Margus Punab ${ }^{270}$, Mostafa Qorbani ${ }^{364}$, Tran Quoc Bao ${ }^{365}$, Ricardas Radisauskas ${ }^{82}$, Salar Rahimikazerooni ${ }^{174}$, Olli Raitakari ${ }^{342}$, Sudha Ramachandra $\operatorname{RaO}^{256}$, Ambady Ramachandran ${ }^{366}$, Elisabete Ramos ${ }^{64}$, $\operatorname{Rafel~Ramos}^{367}$, Lekhraj Rampal ${ }^{368}$, Sanjay Rampal ${ }^{369}$, Josep Redon ${ }^{370}$, Paul Ferdinand M. Reganit ${ }^{371}$, Luis Revilla ${ }^{293}$, Abbas Rezaianzadeh ${ }^{174}$, Robespierre Ribeiro ${ }^{372,448}$, Adrian Richter ${ }^{338}$, Fernando Rigo ${ }^{373}$, Tobias F. Rinke de $\mathrm{Wit}^{374}$, Fernando Rodríguez-Artalejo ${ }^{69}$, María del Cristo Rodriguez-Perez ${ }^{375}$, Laura A. Rodríguez-Villamizar ${ }^{376}$, Ulla Roggenbuck ${ }^{165}$, Rosalba Rojas-Martinez ${ }^{197}$, Dora Romaguera ${ }^{137}$, Elisabetta L. Romeo ${ }^{377}$, Annika Rosengren ${ }^{90,378}$, Joel G. R. Roy ${ }^{131}$, Adolfo Rubinstein ${ }^{8}$, 
Jean-Bernard Ruidavets ${ }^{379}$, Blanca Sandra Ruiz-Betancourt ${ }^{30}$, Paola Russo $^{380}$, Petra Rust ${ }^{268}$, Marcin Rutkowski ${ }^{68}$, Charumathi Sabanayagam ${ }^{204}$, Harshpal S. Sachdev ${ }^{381}$, Alireza Sadjadi ${ }^{4}$, Ali Reza Safarpour ${ }^{174}$, Saeid Safiri ${ }^{172}$, Olfa Saidi ${ }^{382}$, Nader Saki ${ }^{123}$, Benoit Salanave ${ }^{146}$, Diego Salmerón ${ }^{228}$, Veikko Salomaa ${ }^{20}$, Jukka T. Salonen ${ }^{167}$, Massimo Salvetti ${ }^{333}$, Jose Sánchez-Abanto ${ }^{383}$, Susana Sans ${ }^{384}$, Alba M. Santaliestra-Pasías ${ }^{233}$, Diana A. Santos ${ }^{385}$, Maria Paula Santos ${ }^{113}$, Rute Santos ${ }^{113}$, Jouko L. Saramies ${ }^{386}$, Luis B. Sardinha ${ }^{385}$, Nizal Sarrafzadegan $^{387}$, Kai-Uwe Saum ${ }^{105}$, Savvas C. Savva ${ }^{116}$, Norie Sawada ${ }^{388}$, Mariana Sbaraini ${ }^{139}$, Marcia Scazufca ${ }^{389}$, Beatriz D. Schaan ${ }^{139}$, Herman Schargrodsky ${ }^{390}$, Christa Scheidt-Nave $^{107}$, Anja Schienkiewitz ${ }^{107}$, Sabine Schipf ${ }^{338}$, Carsten O. Schmidt ${ }^{338}$, Ben Schöttker ${ }^{105}$, Sara Schramm $^{165}$, Sylvain Sebert ${ }^{61}$, Aye Aye Sein ${ }^{227}$, Abhijit Sen ${ }^{391}$, Sadaf G. Sepanlou ${ }^{4}$, Jennifer Servais ${ }^{131}$, Ramin Shakeri ${ }^{4}$, Svetlana A. Shalnova ${ }^{144}$, Teresa Shamah-Levy ${ }^{197}$, Maryam Sharafkhah ${ }^{4}$, Sanjib K. Sharma ${ }^{190}$, Jonathan E. Shaw ${ }^{301}$, Amaneh Shayanrad ${ }^{4}$, Zumin Shi ${ }^{28}$, Kenji Shibuya ${ }^{392}$, Hana Shimizu-Furusawa ${ }^{393}$, Dong Wook Shin ${ }^{394}$, Youchan Shin ${ }^{204}$, Majid Shirani $^{38}$, Rahman Shiri ${ }^{395}$, Namuna Shrestha ${ }^{88}$, Khairil Si-Ramlee ${ }^{347}$, Alfonso Siani ${ }^{380}$ Rosalynn Siantar ${ }^{204}$, Abla M. Sibai ${ }^{231}$, Diego Augusto Santos Silva ${ }^{141}$, Mary Simon ${ }^{366}$, Judith Simons $^{396}$, Leon A. Simons ${ }^{397}$, Michael Sjöström ${ }^{398}$, Tea Skaaby ${ }^{399}$, Jolanta Slowikowska-Hilczer ${ }^{67}$, Przemyslaw Slusarczyk ${ }^{330}$, Liam Smeeth ${ }^{400}$, Marieke B. Snijder ${ }^{35}$, Stefan Söderberg ${ }^{179}$, Agustinus Soemantri ${ }^{401}$, Reecha Sofat ${ }^{118}$, Vincenzo Solfrizzi ${ }^{402}$, Mohammad Hossein Somi ${ }^{172}$, Emily Sonestedt ${ }^{193}$, Thorkild I. A. Sørensen ${ }^{403}$, Charles Sossa Jérome ${ }^{404}$, Aïcha Soumaré ${ }^{405}$, Kaan Sozmen ${ }^{406}$, Karen Sparrenberger ${ }^{139}$, Jan A. Staessen ${ }^{407}$, Maria G. Stathopoulou ${ }^{408}$, Bill Stavreski ${ }^{244}$, Jostein Steene-Johannessen ${ }^{51}$, Peter Stehle ${ }^{409}$, Aryeh D. Stein ${ }^{308}$, Jochanan Stessman ${ }^{239}$, Ranko Stevanović ${ }^{410}$, Jutta Stieber ${ }^{152,448}$, Doris Stöck ${ }^{152}$, Jakub Stokwiszewski ${ }^{411}$, Karien Stronks ${ }^{35}$, Maria Wany Strufaldi ${ }^{180}$, Ramón Suárez-Medina ${ }^{412}$, Chien-An Sun ${ }^{413}$, Johan Sundström ${ }^{291}$, Paibul Suriyawongpaisal ${ }^{14}$, Rody G $\mathrm{Sy}^{371}$, René Charles Sylva ${ }^{414}$, Moyses Szklo ${ }^{255}$, E. Shyong Tai ${ }^{283}$, Abdonas Tamosiunas ${ }^{82}$, Eng Joo Tan $^{76}$, Mohammed Rasoul Tarawneh ${ }^{415}$, Carolina B. Tarqui-Mamani ${ }^{383}$, Anne Taylor ${ }^{192}$, Julie Taylor ${ }^{18}$, Grethe S. Tell ${ }^{214}$, Tania Tello ${ }^{81}$, K. R. Thankappan ${ }^{416}$, Lutgarde Thijs ${ }^{407}$, Betina H. Thuesen ${ }^{26}$, Ulla Toft ${ }^{26}$, Hanna K. Tolonen ${ }^{20}$, Janne S. Tolstrup ${ }^{89}$, Murat Topbas ${ }^{166}$, Roman Topór-Madry $^{201}$, María José Tormo ${ }^{417}$, Michael J. Tornaritis ${ }^{116}$, Maties Torrent ${ }^{418}$, Laura Torres-Collado ${ }^{186}$, Pierre Traissac ${ }^{303}$, Oanh T. H. Trinh ${ }^{339}$, Julia Truthmann ${ }^{107}$, Shoichiro Tsugane $^{388}$, Marshall K. Tulloch-Reid ${ }^{162}$, Tomi-Pekka Tuomainen ${ }^{212}$, Jaakko Tuomilehto ${ }^{20}$, Anne Tybjaerg-Hansen ${ }^{24}$, Christophe Tzourio ${ }^{405}$, Peter Ueda ${ }^{398}$, Eunice Ugel ${ }^{419}$, Hanno Ulmer $^{263}$, Belgin Unal ${ }^{420}$, Hannu M. T. Uusitalo ${ }^{421}$, Gonzalo Valdivia ${ }^{22}$, Damaskini Valvi ${ }^{422}$, Rob M. van Dam ${ }^{283}$, Yvonne T. van der Schouw ${ }^{423}$, Koen Van Herck ${ }^{132}$, Hoang Van Minh ${ }^{424}$, Lenie van Rossem ${ }^{425}$, Natasja M. Van Schoor ${ }^{229}$, Irene G. M. van Valkengoed ${ }^{35}$, Dirk Vanderschueren $^{130}$, Diego Vanuzzo ${ }^{360}$, Anette Varbo ${ }^{24}$, Patricia Varona-Pérez ${ }^{412}$, Senthil K. Vasan $^{135}$, Lars Vatten ${ }^{240}$, Tomas Vega ${ }^{294}$, Toomas Veidebaum ${ }^{292}$, Gustavo Velasquez-Melendez ${ }^{188}$, Silvia J. Venero-Fernández ${ }^{412}$, Giovanni Veronesi ${ }^{191}$, W. M. Monique Verschuren $^{92}$, Cesar G. Victora ${ }^{60}$, Dhanasari Vidiawati ${ }^{426}$, Lucie Viet ${ }^{92}$, Salvador Villalpando $^{197}$, Jesus Vioque ${ }^{427}$, Jyrki K. Virtanen ${ }^{212}$, Sophie Visvikis-Siest ${ }^{408}$, Bharathi Viswanathan ${ }^{101}$, Tiina Vlasoff ${ }^{428}$, Peter Vollenweider ${ }^{307}$, Ari Voutilainen ${ }^{212}$, Alisha N. Wade ${ }^{429}$, Aline Wagner ${ }^{323}$, Janette Walton ${ }^{430}$, Wan Mohamad Wan Bebakar ${ }^{178}$, Wan Nazaimoon Wan Mohamud $^{431}$, Ming-Dong Wang ${ }^{432}$, Ningli Wang ${ }^{433}$, Qian Wang ${ }^{434}$, Ya Xing Wang ${ }^{435}$, Ying-Wei Wang ${ }^{125}$, S. Goya Wannamethee ${ }^{118}$, Niels Wedderkopp ${ }^{202}$, Wenbin Wei ${ }^{435}$, Peter H. Whincup $^{436}$, Kurt Widhalm ${ }^{437}$, Indah S. Widyahening ${ }^{426}$, Andrzej Wiecek ${ }^{129}$, Alet H. Wijga ${ }^{92}$, Rainford J. Wilks ${ }^{162}$, Johann Willeit ${ }^{263}$, Peter Willeit ${ }^{263}$, Tom Wilsgaard ${ }^{310}$, Bogdan Wojtyniak ${ }^{411}$, Roy A. Wong-McClure ${ }^{27}$, Andrew Wong ${ }^{118}$, Tien Yin Wong ${ }^{122}$, Jean Woo ${ }^{220}$, Mark Woodward ${ }^{397,438}$, Frederick C. Wu ${ }^{344}$, Shouling Wu ${ }^{121}$, Haiquan $\mathrm{Xu}^{439}$, Liang Xu${ }^{433}$, Weili Yan ${ }^{440}$, Xiaoguang Yang ${ }^{216}$, Tabara Yasuharu ${ }^{183}$, Xingwang Ye ${ }^{290}$, Toh Peng Yeow ${ }^{441}$, Panayiotis K. Yiallouros ${ }^{442}$, Moein Yoosefi ${ }^{4}$, Akihiro Yoshihara ${ }^{238}$, San-Lin You ${ }^{413}$, Novie O. Younger-Coleman ${ }^{162}$, Ahmad Faudzi Yusoff ${ }^{37}$, Ahmad A. Zainuddin ${ }^{37}$, Seyed Rasoul Zakavi ${ }^{168}$ Mohammad Reza Zali ${ }^{6}$, Farhad Zamani ${ }^{443}$, Sabina Zambon ${ }^{306}$, Antonis Zampelas ${ }^{302}$, Ko Ko $Z_{Z^{2}}{ }^{282}$, Tomasz Zdrojewski ${ }^{68}$, Tajana Zeljkovic Vrkic ${ }^{242}$, Zhen-Yu Zhang ${ }^{407}$, Wenhua Zhao ${ }^{216}$, Shiqi Zhen ${ }^{444}$, Yingfeng Zheng ${ }^{445}$, Bekbolat Zholdin ${ }^{446}$, Baurzhan Zhussupov ${ }^{80}$, Nada Zoghlami $^{54}$, Julio Zuñiga Cisneros ${ }^{331}$, Edward W. Gregg ${ }^{1}$ \& Majid Ezzati ${ }^{1447 凶}$

${ }^{1}$ Imperial College London, London, UK. ${ }^{2}$ Harvard T. H. Chan School of Public Health, Boston, MA, USA. ${ }^{3}$ University of Auckland, Auckland, New Zealand. ${ }^{4}$ Tehran University of Medical Sciences, Tehran, Iran. ${ }^{5}$ Middlesex University, London, UK. ${ }^{6}$ Shahid Beheshti University of Medical Sciences, Tehran, Iran. ${ }^{7}$ Rush University Medical Center, Chicago, IL, USA. ${ }^{8}$ Institute for Clinical Effectiveness and Health Policy, Buenos Aires, Argentina. ${ }^{9} \mathrm{H}$ arvard Medical School, Boston, MA, USA. ${ }^{10}$ Erasmus Medical Center Rotterdam, Rotterdam, The Netherlands ${ }^{11}$ World Health Organization, Geneva, Switzerland. ${ }^{12}$ Independent researcher, Los Angeles, CA, USA. ${ }^{13}$ University of California Berkeley, Berkeley, CA, USA. ${ }^{14}$ Mahidol University, Nakhon Pathom, Thailand. ${ }^{15}$ Charles University in Prague, Prague, Czech Republic. ${ }^{16}$ Thomayer Hospital, Prague, Czech Republic. ${ }^{17}$ Istituto Superiore di Sanità, Rome, Italy. ${ }^{18}$ South African Medical Research Council, Cape Town, South Africa. ${ }^{19}$ Seoul National University, Seoul, Republic of Korea. ${ }^{20}$ Finnish Institute for Health and Welfare, Helsinki, Finland. ${ }^{21} \mathrm{ICMR}-$ National Institute of Nutrition, Hyderabad, India. ${ }^{22}$ Pontificia Universidad Católica de Chile, Santiago, Chile. ${ }^{23}$ ICMR-National Centre for Disease Informatics and Research, Bengaluru, India. ${ }^{24}$ Copenhagen University Hospital, Copenhagen, Denmark. ${ }^{25}$ Capital Medical University Beijing An Zhen Hospital, Beijing, China. ${ }^{26}$ Bispebjerg and Frederiksberg Hospital, Copenhagen, Denmark. ${ }^{27}$ Caja Costarricense de Seguro Social, San José, Costa Rica. ${ }^{28}$ Qatar University, Doha, Qatar. ${ }^{29}$ Birzeit University, Birzeit, Palestine. ${ }^{30}$ Instituto Mexicano del Seguro Social, Mexico City, Mexico. ${ }^{31}$ Flinders University, Adelaide, South Australia, Australia. ${ }^{32}$ Food and Nutrition Research Institute, Taguig, The Philippines. ${ }^{33}$ Urmia University of Medical Sciences, Urmia, Iran. ${ }^{34}$ Instituto Nacional de Ciencias Médicas y Nutricion, Mexico City,
Mexico. ${ }^{35}$ University of Amsterdam, Amsterdam, The Netherlands. ${ }^{36}$ Steno Diabetes Center Copenhagen, Gentofte, Denmark. ${ }^{37}$ Ministry of Health Malaysia, Kuala Lumpur, Malaysia. ${ }^{38}$ Shahrekord University of Medical Sciences, Shahrekord, Iran. ${ }^{39}$ University of Oslo, Oslo, Norway. ${ }^{40}$ University of Bremen, Bremen, Germany. ${ }^{41}$ National Center for Diabetes, Endocrinology and Genetics, Amman, Jordan. ${ }^{42}$ Dasman Diabetes Institute, Kuwait City, Kuwait. ${ }^{43}$ Aldara Hospital and Medical Center, Riyadh, Saudi Arabia. ${ }^{44}$ King Abdullah International Medical Research Center, Riyadh, Saudi Arabia. ${ }^{45}$ Luxembourg Institute of Health, Strassen, Luxembourg. ${ }^{46}$ World Health Organization Regional Office for the Eastern Mediterranean, Cairo, Egypt. ${ }^{47}$ Bombay Hospital and Medical Research Centre, Mumbai, India. ${ }^{48}$ University of Lille, Lille, France. ${ }^{49}$ Lille University Hospital, Lille, France. ${ }^{50}$ Western Norway University of Applied Sciences, Sogndal, Norway. ${ }^{51}$ Norwegian School of Sport Sciences, Oslo, Norway. ${ }^{52}$ Madras Diabetes Research Foundation, Chennai, India. ${ }^{53}$ Zahedan University of Medical Sciences, Zahedan, Iran. ${ }^{54}$ National Institute of Public Health, Tunis, Tunisia. ${ }^{55}$ Institute of Public Health of the University of Porto, Porto, Portugal. ${ }^{56}$ Norwegian Institute of Public Health, Oslo, Norway. ${ }^{57}$ University of Massachusetts, Amherst, MA, USA. ${ }^{58} \mathrm{Abt}$ Associates, Kathmandu, Nepal. ${ }^{59}$ University of Iceland, Reykjavik, Iceland. ${ }^{60}$ Federal University of Pelotas, Pelotas, Brazil. ${ }^{61}$ University of Oulu, Oulu, Finland. ${ }^{62}$ Oulu University Hospital, Oulu, Finland. ${ }^{63}$ Regional Authority of Public Health, Banska Bystrica, Slovakia. ${ }^{64}$ University of Porto Medical School, Porto, Portugal. ${ }^{65}$ Research Institute for Endocrine Sciences, Tehran, Iran. ${ }^{66}$ University of Science and Technology, Sana'a, Yemen. ${ }^{67}$ Medical University of Lodz, Lodz, Poland. ${ }^{68}$ Medical University of Gdansk, Gdansk, Poland. ${ }^{69}$ Universidad Autónoma de Madrid/ CIBERESP, Madrid, Spain. ${ }^{70}$ University of Palermo, Palermo, Italy. ${ }^{71}$ Pan American Health Organization, Washington, DC, USA. ${ }^{72}$ Mohammed V University de Rabat, Rabat, Morocco. ${ }^{73}$ Dalhousie University, Halifax, Nova Scotia, Canada. ${ }^{74}$ Jordan University of Science and Technology, Irbid, Jordan. ${ }^{75} \mathrm{Al}$-Farabi Kazakh National University, Almaty, Kazakhstan. ${ }^{76}$ University of Sydney, Sydney, New South Wales, Australia. ${ }^{77}$ Christian Medical College, Vellore, India. ${ }^{78}$ University Tunis El Manar, Tunis, Tunisia. ${ }^{79} \mathrm{Cafam}$ University Foundation, Bogota, Colombia. ${ }^{80}$ Kazakh National Medical University, Almaty, Kazakhstan. ${ }^{81}$ Universidad Peruana Cayetano Heredia, Lima, Peru. ${ }^{82}$ Lithuanian University of Health Sciences, Kaunas, Lithuania. ${ }^{83}$ University of São Paulo, São Paulo, Brazil. ${ }^{84}$ Sunder Lal Jain Hospital, Delhi, India. ${ }^{85}$ Shanghai Jiao-Tong University School of Medicine, Shanghai, China. ${ }^{86}$ Public Health Agency of Canada, Ottawa, Ontario, Canada. ${ }^{87}$ Ufa Eye Research Institute, Ufa, Russia. ${ }^{88} \mathrm{Nepal} \mathrm{Health}$ Research Council, Kathmandu, Nepal. ${ }^{89}$ University of Southern Denmark, Copenhagen, Denmark. ${ }^{90}$ University of Gothenburg, Gothenburg, Sweden. ${ }^{91}$ Universidade Federal do Rio de Janeiro, Rio de Janeiro, Brazil. ${ }^{92}$ National Institute for Public Health and the Environment, Bilthoven, The Netherlands. ${ }^{93}$ University of Turin, Turin, Italy. ${ }^{94}$ Nanyang Technological University, Singapore, Singapore. ${ }^{95}$ Universidad de la República, Montevideo, Uruguay. ${ }^{96}$ Centro de Educación Médica e Investigaciones Clínicas, Buenos Aires, Argentina. ${ }^{97}$ IRCCS Neuromed, Pozzilli, Italy. ${ }^{98}$ Toulouse University School of Medicine, Toulouse, France. ${ }^{99}$ University Hospital of Varese, Varese, Italy. ${ }^{100}$ University Hospital KU Leuven, Leuven, Belgium. ${ }^{101}$ Ministry of Health, Victoria, Seychelles. ${ }^{102}$ University of Lausanne, Lausanne, Switzerland. ${ }^{103}$ Universidad Central de Venezuela, Caracas, Venezuela. ${ }^{104}$ Bielefeld University, Bielefeld, Germany. ${ }^{105}$ German Cancer Research Center, Heidelberg, Germany. ${ }^{106}$ University College Copenhagen, Copenhagen, Denmark. ${ }^{107}$ Robert Koch Institute, Berlin, Germany. ${ }^{108}$ Universidad de La Laguna, Tenerife, Spain. ${ }^{109}$ University of Malta, Msida, Malta. ${ }^{110}$ Istanbul University - Cerrahpasa, Istanbul, Turkey. ${ }^{11}$ Universidade Federal de Juiz de Fora, Juiz de Fora, Brazil. ${ }^{12}$ Gaetano Fucito Hospital, Mercato San Severino, Italy. ${ }^{113}$ University of Porto, Porto, Portugal. ${ }^{114}$ Santiago de Compostela University, Santiago, Spain. ${ }^{115}$ Council for Agricultural Research and Economics, Rome, Italy. ${ }^{116}$ Research and Education Institute of Child Health, Nicosia, Cyprus. ${ }^{177}$ Dr. A. Ramachandran's Diabetes Hospital, Chennai, India. ${ }^{118}$ University College London, London, UK. ${ }^{119}$ Academia Sinica, Taipei, Taiwan. ${ }^{120}$ Capital Institute of Pediatrics, Beijing, China. ${ }^{121}$ Kailuan General Hospital, Tangshan, China. ${ }^{122}$ Duke-NUS Medical School, Singapore, Singapore. ${ }^{123}$ Ahvaz Jundishapur University of Medical Sciences, Ahvaz, Iran. ${ }^{124}$ The Gertner Institute for Epidemiology and Health Policy Research, Ramat Gan, Israel. ${ }^{125}$ Ministry of Health and Welfare, Taipei, Taiwan. ${ }^{126}$ Murcia Health Council, Murcia, Spain. ${ }^{127}$ Seoul National University College of Medicine, Seoul, Republic of Korea. ${ }^{128} \mathrm{Korea}$ Centers for Disease Control and Prevention, Cheongju-si, Republic of Korea. ${ }^{129}$ Medical University of Silesia, Katowice, Poland. ${ }^{130}$ Katholieke Universiteit Leuven, Leuven, Belgium. ${ }^{131}$ Statistics Canada, Ottawa, Ontario, Canada. ${ }^{132}$ Ghent University, Ghent, Belgium. ${ }^{133}$ Agency for Preventive and Social Medicine, Bregenz, Austria. ${ }^{134}$ Federal University of Maranhão, São Luís, Brazil. ${ }^{135}$ University of Southampton, Southampton, UK. ${ }^{136}$ Institut Pasteur de Lille, Lille, France. ${ }^{137}$ CIBEROBN, Madrid, Spain. ${ }^{138}$ University of Debrecen, Debrecen, Hungary. ${ }^{139}$ Universidade Federal do Rio Grande do Sul, Porto Alegre, Brazil. ${ }^{140}$ National Council of Research, Reggio Calabria, Italy. ${ }^{141}$ Federal University of Santa Catarina, Florianópolis, Brazil. ${ }^{142}$ Eduardo Mondlane University, Maputo, Mozambique. ${ }^{143}$ Geneva University Hospitals, Geneva, Switzerland. ${ }^{144}$ National Research Centre for Preventive Medicine, Moscow, Russia. ${ }^{145}$ University of Montreal, Montreal, Québec, Canada. ${ }^{146}$ French Public Health Agency, St Maurice, France. ${ }^{147}$ Mediterranea Cardiocentro, Naples, Italy. ${ }^{148}$ University of Zagreb, Zagreb, Croatia. ${ }^{149}$ Ministry of Health and Medical Education, Tehran, Iran. ${ }^{150}$ University of Queensland, Brisbane, Queensland, Australia. ${ }^{151}$ Universidad de Cuenca, Cuenca, Ecuador. ${ }^{152} \mathrm{Helmholtz}$ Zentrum München, Munich, Germany. ${ }^{153}$ Carol Davila University of Medicine and Pharmacy, Bucharest, Romania. ${ }^{154}$ University Hospital Düsseldorf, Düsseldorf, Germany. ${ }^{155}$ National Institute of Cardiology, Warsaw, Poland. ${ }^{156}$ Beth Israel Deaconess Medical Center, Boston, MA, USA. ${ }^{157}$ University of Latvia, Riga, Latvia. ${ }^{158}$ Ministry of Health and Medical Services, Gizo, Solomon Islands. ${ }^{159}$ Hormozgan University of Medical Sciences, Bandar Abbas, Iran. ${ }^{160}$ University of Skövde, Skövde, Sweden. ${ }^{161}$ National Institute of Nutrition and Food Technology, Tunis, Tunisia. ${ }^{162}$ The University of the West Indies, Kingston, Jamaica. ${ }^{163}$ Institut 


\section{Article}

Hospital del Mar d'Investigacions Mèdiques, Barcelona, Spain. ${ }^{164}$ University of Stellenbosch, Cape Town, South Africa. ${ }^{165}$ University of Duisburg-Essen, Duisburg, Germany. ${ }^{166} \mathrm{Karadeniz}$ Technical University, Trabzon, Turkey. ${ }^{167}$ University of Helsinki, Helsinki, Finland. ${ }^{168}$ Mashhad University of Medical Sciences, Mashhad, Iran. ${ }^{169}$ Rafsanjan University of Medical Sciences, Rafsanjan, Iran. ${ }^{170}$ Queen's University of Belfast, Belfast, UK. ${ }^{17}$ University of Zurich, Zurich, Switzerland. ${ }^{172}$ Tabriz University of Medical Sciences, Tabriz, Iran. ${ }^{173}$ Fasa University of Medical Sciences, Fasa, Iran. ${ }^{174}$ Shiraz University of Medical Sciences, Shiraz, Iran. ${ }^{175}$ Centro de Salud Villanueva Norte, Badajoz, Spain. ${ }^{176}$ Servicio Extremeño de Salud, Badajoz, Spain. ${ }^{177}$ Ministry of Health, Buenos Aires, Argentina. ${ }^{178}$ Universiti Sains Malaysia, Kelantan, Malaysia. ${ }^{179}$ Umeå University, Umeå, Sweden. ${ }^{180}$ Federal University of São Paulo, São Paulo, Brazil. ${ }^{181} \mathrm{Hospital}$ Universitario Son Espases, Palma, Spain. ${ }^{182}$ Kindai University, Osaka-Sayama, Japan. ${ }^{183} \mathrm{Kyoto}$ University, Kyoto, Japan. ${ }^{184}$ Medical University of Warsaw, Warsaw, Poland. ${ }^{185}$ University of Catania, Catania, Italy. ${ }^{186} \mathrm{CIBER}$ en Epidemiología y Salud Pública, Alicante, Spain. ${ }^{187} \mathrm{CIBER}$ en Epidemiología y Salud Pública, Barcelona, Spain. ${ }^{18}$ Universidade Federal de Minas Gerais, Belo Horizonte, Brazil. ${ }^{199}$ Wageningen University, Wageningen, The Netherlands. ${ }^{190}$ B. P. Koirala Institute of Health Sciences, Dharan, Nepal. ${ }^{191}$ University of Insubria, Varese, Italy. ${ }^{192}$ University of Adelaide, Adelaide, South Australia, Australia. ${ }^{193}$ Lund University, Lund, Sweden. ${ }^{194} \mathrm{McGill}$ University, Montreal, Québec, Canada. ${ }^{195}$ Universidad Politécnica de Madrid, Madrid, Spain. ${ }^{196}$ St Anne's University Hospital, Brno, Czech Republic. ${ }^{197}$ National Institute of Public Health, Cuernavaca, Mexico. ${ }^{198}$ Centro de Estudios en Diabetes A.C., Mexico City, Mexico. ${ }^{199}$ Universidad Autónoma de Santo Domingo, Santo Domingo, Dominican Republic. ${ }^{200}$ Institute for Clinical and Experimental Medicine, Prague, Czech Republic. ${ }^{201}$ Jagiellonian University Medical College, Kraków, Poland. ${ }^{202}$ University of Southern Denmark, Odense, Denmark. ${ }^{203}$ National Center of Cardiovascular Diseases, Beijing, China. ${ }^{204}$ Singapore Eye Research Institute, Singapore, Singapore. ${ }^{205}$ Icelandic Heart Association, Kopavogur, Iceland. ${ }^{206}$ Universidad Icesi, Cali, Colombia. ${ }^{207} \mathrm{Eternal}$ Heart Care Centre and Research Institute, Jaipur, India. ${ }^{208}$ National Health Insurance Service, Wonju, Republic of Korea. ${ }^{209}$ Prevention of Metabolic Disorders Research Center, Tehran, Iran. ${ }^{210}$ The University of the West Indies, Cave Hill, Barbados. ${ }^{211}$ Kermanshah University of Medical Sciences, Kermanshah, Iran. ${ }^{212}$ University of Eastern Finland, Kuopio, Finland. ${ }^{213}$ Kyushu University, Fukuoka, Japan. ${ }^{214}$ University of Bergen, Bergen, Norway. ${ }^{215}$ Tulane University, New Orleans, LA, USA. ${ }^{216}$ Chinese Center for Disease Control and Prevention, Beijing, China. ${ }^{27} \mathrm{~J}$ Joep Lange Institute, Amsterdam, The Netherlands. ${ }^{218} \mathrm{Chronic}$ Diseases Research Center, Tehran, Iran. ${ }^{219}$ University of Hong Kong, Hong Kong, China. ${ }^{220}$ The Chinese University of Hong Kong, Hong Kong, China. ${ }^{221}$ University of Western Australia, Perth, Western Australia, Australia. ${ }^{222}$ Kingston Health Sciences Centre, Kingston, Ontario, Canada. ${ }^{223} \mathrm{Heart}$ Institute, São Paulo, Brazil. ${ }^{224} \mathrm{Fundación}$ Oftalmológica de Santander, Bucaramanga, Colombia. ${ }^{225}$ University Oran 1, Oran, Algeria. ${ }^{226}$ Independent Public Health Specialist, Nay Pyi Taw, Myanmar. ${ }^{227}$ Ministry of Health and Sports, Nay Pyi Taw, Myanmar. ${ }^{228} \mathrm{ClBER}$ en Epidemiología y Salud Pública, Murcia, Spain. ${ }^{229} \mathrm{VU}$ University Medical Center, Amsterdam, The Netherlands. ${ }^{230}$ International Agency for Research on Cancer, Lyon, France. ${ }^{231}$ American University of Beirut, Beirut, Lebanon. ${ }^{232}$ Cairo University, Cairo, Egypt. ${ }^{233}$ University of Zaragoza, Zaragoza, Spain. ${ }^{234}$ National Institutes of Biomedical Innovation, Health and Nutrition, Tokyo, Japan. ${ }^{235}$ Medical University Varna, Varna, Bulgaria. ${ }^{236}$ The University of Tokyo, Tokyo, Japan. ${ }^{237}$ The Hospital for Sick Children, Toronto, Ontario, Canada. ${ }^{238}$ Niigata University, Niigata, Japan. ${ }^{239}$ Hadassah University Medical Center, Jerusalem, Israel. ${ }^{240}$ Norwegian University of Science and Technology, Trondheim, Norway. ${ }^{241}$ University of Melbourne, Melbourne, Victoria, Australia. ${ }^{242}$ University Hospital Centre Zagreb, Zagreb, Croatia. ${ }^{243}$ University of Zagreb School of Medicine, Zagreb, Croatia. ${ }^{244} \mathrm{Heart}$ Foundation, Melbourne, Victoria, Australia. ${ }^{245}$ Guangzhou 12th Hospital, Guangzhou, China. ${ }^{246}$ Universidad Eugenio Maria de Hostos, Santo Domingo, Dominican Republic. ${ }^{24}$ Simon Fraser University, Burnaby, British Columbia, Canada. ${ }^{248}$ Ruprecht-Karls-University of Heidelberg, Heidelberg, Germany. ${ }^{249}$ World Health Organization Country Office, Delhi, India. ${ }^{250}$ Guilan University of Medical Sciences, Rasht, Iran. ${ }^{251}$ University of Opole, Opole, Poland. ${ }^{252}$ University of Crete, Heraklion, Greece. ${ }^{253}$ Universiti Kebangsaan Malaysia, Kuala Lumpur, Malaysia. ${ }^{254}$ Maharajgunj Medical Campus, Kathmandu, Nepal. ${ }^{255}$ Johns Hopkins Bloomberg School of Public Health, Baltimore, MD, USA. ${ }^{256}$ National Institute of Epidemiology, Chennai, India. ${ }^{257}$ University of Münster, Münster, Germany. ${ }^{258}$ Research Institute for Primordial Prevention of Non-communicable Disease, Isfahan, Iran. ${ }^{25} \mathrm{Kyrgyz}$ State Medical Academy, Bishkek, Kyrgyzstan. ${ }^{260}$ Research Institute of Child Nutrition, Dortmund, Germany. ${ }^{261}$ Mazandaran University of Medical Sciences, Sari, Iran. ${ }^{262} \mathrm{Hypertension}$ Research Center, Isfahan, Iran. ${ }^{263}$ Medical University of Innsbruck, Innsbruck, Austria. ${ }^{264}$ Muhimbili University of Health and Allied Sciences, Dar es Salaam, Tanzania. ${ }^{265}$ Yonsei University College of Medicine, Seoul, Republic of Korea. ${ }^{266}$ National Cancer Center, Goyang-si, Republic of Korea. ${ }^{267}$ University of Pennsylvania, Philadelphia, PA, USA. ${ }^{268}$ University of Vienna, Vienna, Austria. ${ }^{269}$ Oulu Deaconess Institute Foundation, Oulu, Finland. ${ }^{270}$ Tartu University Clinics, Tartu, Estonia. ${ }^{271} \mathrm{Kansai}$ Medical University, Hirakata, Japan. ${ }^{272}$ Ministry of Health and Quality of Life, Port Louis, Mauritius. ${ }^{273}$ University Hospital Ulm, Ulm, Germany. ${ }^{274}$ University of Groningen, Groningen, The Netherlands. ${ }^{275}$ University of Jyväskylä, Jyväskylä, Finland. ${ }^{276}$ African Population and Health Research Center, Nairobi, Kenya. ${ }^{27}$ Higher Institute of Health Sciences of Settat, Settat, Morocco. ${ }^{278}$ Ministry of Health, Algiers, Algeria. ${ }^{279}$ Harokopio University, Athens, Greece. ${ }^{280}$ Sahlgrenska Academy, Gothenburg, Sweden. ${ }^{281}$ Endocrinology and Metabolism Research Center, Tehran, Iran. ${ }^{282}$ University of Public Health, Yangon, Myanmar. ${ }^{283}$ National University of Singapore, Singapore, Singapore. ${ }^{284}$ Tampere University Hospital, Tampere, Finland. ${ }^{285}$ Tampere University, Tampere, Finland. ${ }^{286}$ University of Cape Town, Cape Town, South Africa. ${ }^{287}$ West Virginia University, Morgantown, WV, USA. ${ }^{288}$ Oswaldo Cruz Foundation Rene Rachou Research Institute, Belo Horizonte, Brazil. ${ }^{289}$ National Taiwan University, Taipei, Taiwan. ${ }^{200}$ University of Chinese Academy of Sciences, Shanghai, China.
${ }^{291}$ Uppsala University, Uppsala, Sweden. ${ }^{292}$ National Institute for Health Development, Tallinn, Estonia. ${ }^{293}$ Universidad San Martín de Porres, Lima, Peru. ${ }^{294}$ Consejería de Sanidad Junta de Castilla y León, Valladolid, Spain. ${ }^{295}$ Norrbotten County Council, Luleå, Sweden. ${ }^{296}$ Peking University, Beijing, China. ${ }^{207}$ Universidade Federal de Ouro Preto, Ouro Preto, Brazil. ${ }^{298}$ University of Coimbra, Coimbra, Portugal. ${ }^{299}$ The Jikei University School of Medicine, Tokyo, Japan. ${ }^{300}$ Institute of Neuroscience of the National Research Council, Padua, Italy. ${ }^{301}$ Baker Heart and Diabetes Institute, Melbourne, Victoria, Australia. ${ }^{302}$ Agricultural University of Athens, Athens, Greece. ${ }^{303}$ French National Research Institute for Sustainable Development, Montpellier, France. ${ }^{304} \mathrm{Hospital}$ Israelita Albert Einstein, São Paulo, Brazil. ${ }^{305}$ University of Otago, Dunedin, New Zealand. ${ }^{306}$ University of Padua, Padua, Italy. ${ }^{307}$ Lausanne University Hospital, Lausanne, Switzerland. ${ }^{308}$ Emory University, Atlanta, GA, USA. ${ }^{309}$ Universidade Estadual do Centro-Oeste, Guarapuava, Brazil. ${ }^{310} \mathrm{UiT}$ The Arctic University of Norway, Troms $\varnothing$, Norway. ${ }^{311}$ Cape Peninsula University of Technology, Cape Town, South Africa. ${ }^{312}$ Brown University, Providence, RI, USA. ${ }^{313}$ University of Edinburgh, Edinburgh, UK. ${ }^{314}$ University College Dublin, Dublin, Ireland. ${ }^{315}$ Shahid Sadoughi University of Medical Sciences, Yazd, Iran. ${ }^{316}$ Institut National de la Santé et de la Recherche Médicale, Lille, France. ${ }^{37}$ ICMR-National Institute of Medical Statistics, New Delhi, India. ${ }^{318}$ Lusófona University, Lisbon, Portugal. ${ }^{319}$ Women's Social and Health Studies Foundation, Trivandrum, India. ${ }^{220}$ Università degli Studi di Firenze, Florence, Italy. ${ }^{321}$ Ain Shams University, Cairo, Egypt. ${ }^{322}$ Isfahan Cardiovascular Research Center, Isfahan, Iran. ${ }^{323}$ University of Strasbourg, Strasbourg, France. ${ }^{324}$ Strasbourg University Hospital, Strasbourg, France. ${ }^{325}$ University of Pécs, Pécs, Hungary. ${ }^{326}$ Mulago Hospital, Kampala, Uganda. ${ }^{327}$ University of Medical Sciences of Cienfuegos, Cienfuegos, Cuba. ${ }^{328}$ Royal College of Surgeons in Ireland Dublin, Dublin, Ireland. ${ }^{329}$ La Trobe University, Melbourne, Victoria, Australia. ${ }^{330}$ International Institute of Molecular and Cell Biology, Warsaw, Poland. ${ }^{331}$ Instituto Conmemorativo Gorgas de Estudios de la Salud, Panama City, Panama. ${ }^{332}$ World Health Organization Country Office, Lilongwe, Malawi. ${ }^{333}$ University of Brescia, Brescia, Italy. ${ }^{334}$ Bushehr University of Medical Sciences, Bushehr, Iran. ${ }^{335} \mathrm{Ulm}$ University, Ulm, Germany. ${ }^{336}$ Kobe University, Kobe, Japan. ${ }^{337}$ Suraj Eye Institute, Nagpur, India. ${ }^{338}$ University Medicine of Greifswald, Greifswald, Germany. ${ }^{339}$ University of Medicine and Pharmacy at Ho Chi Minh City, Ho Chi Minh City, Vietnam. ${ }^{340} \mathrm{Hanoi}$ Medical University, Hanoi, Vietnam. ${ }^{341}$ Miami Veterans Affairs Healthcare System, Miami, FL, USA. ${ }^{342}$ University of Turku, Turku, Finland. ${ }^{343}$ Eastern Mediterranean Public Health Network, Amman, Jordan. ${ }^{344}$ University of Manchester, Manchester, UK. ${ }^{345}$ Japan Wildlife Research Center, Tokyo, Japan. ${ }^{346}$ Istanbul University, Istanbul, Turkey. ${ }^{347}$ Ministry of Health, Bandar Seri Begawan, Brunei. ${ }^{348}$ University of Madeira, Funchal, Portugal. ${ }^{349}$ MRC Lifecourse Epidemiology Unit, Southampton, UK. ${ }^{350}$ University of Novi Sad, Novi Sad, Serbia. ${ }^{351}$ Kwame Nkrumah University of Science and Technology, Kumasi, Ghana. ${ }^{352}$ Institute for Social and Preventive Medicine, Ottawa, Ontario, Canada. ${ }^{353}$ IRCCS Ente Ospedaliero Specializzato in Gastroenterologia S. de Bellis, Bari, Italy. ${ }^{354}$ Jivandeep Hospital, Anand, India. ${ }^{355}$ South African Medical Research Council, Durban, South Africa. ${ }^{356}$ Vietnam National Heart Institute, Hanoi, Vietnam. ${ }^{357}$ Clínica de Medicina Avanzada Dr. Abel González, Santo Domingo, Dominican Republic. ${ }^{358}$ Leibniz Institute for Prevention Research and Epidemiology - BIPS, Bremen, Germany. ${ }^{359}$ University of Sarajevo, Sarajevo, Bosnia and Herzegovina. ${ }^{360}$ Cardiovascular Prevention Centre, Udine, Italy. ${ }^{361}$ Public Health Agency of Catalonia, Barcelona, Spain. ${ }^{362}$ Observatorio de Salud Pública de Santander, Bucaramanga, Colombia. ${ }^{363}$ Ardabil University of Medical Sciences, Ardabil, Iran. ${ }^{364}$ Alborz University of Medical Sciences, Karaj, Iran. ${ }^{365}$ Ministry of Health, Hanoi, Vietnam. ${ }^{366}$ India Diabetes Research Foundation, Chennai, India. ${ }^{367}$ Institut Universitari d'Investigació en Atenció Primària Jordi Gol, Girona, Spain. ${ }^{368}$ Universiti Putra Malaysia, Serdang, Malaysia. ${ }^{369}$ University of Malaya, Kuala Lumpur, Malaysia. ${ }^{370}$ University of Valencia, Valencia, Spain. ${ }^{37}$ University of the Philippines, Manila, The Philippines. ${ }^{372}$ Minas Gerais State Secretariat for Health, Belo Horizonte, Brazil. ${ }^{373} \mathrm{CS}$ S. Agustín Ibsalut, Palma, Spain. ${ }^{374}$ Amsterdam Institute for Global Health and Development, Amsterdam, The Netherlands. ${ }^{375}$ Canarian Health Service, Tenerife, Spain. ${ }^{376}$ Universidad Industrial de Santander, Bucaramanga, Colombia. ${ }^{377}$ Associazione Calabrese di Epatologia, Reggio Calabria, Italy. ${ }^{378}$ Sahlgrenska University Hospital, Gothenburg, Sweden. ${ }^{379}$ Toulouse University Hospital, Toulouse, France. ${ }^{380}$ Institute of Food Sciences of the National Research Council, Avellino, Italy. ${ }^{381}$ Sitaram Bhartia Institute of Science and Research, New Delhi, India. ${ }^{382}$ Faculty of Medicine of Tunis, Tunis, Tunisia. ${ }^{383}$ National Institute of Health, Lima, Peru. ${ }^{384}$ Catalan Department of Health, Barcelona, Spain. ${ }^{385}$ Universidade de Lisboa, Lisbon, Portugal. ${ }^{386}$ South Karelia Social and Health Care District, Lappeenranta, Finland. ${ }^{387}$ Cardiovascular Research Institute, Isfahan, Iran. ${ }^{388}$ National Cancer Center, Tokyo, Japan. ${ }^{389}$ University of São Paulo Clinics Hospital, São Paulo, Brazil. ${ }^{390} \mathrm{Hospital}$ Italiano de Buenos Aires, Buenos Aires, Argentina. ${ }^{391} \mathrm{Center}$ for Oral Health Services and Research Mid-Norway, Trondheim, Norway. ${ }^{392}$ King's College London, London, UK. ${ }^{393} \mathrm{National}$ Center for Global Health and Medicine, Tokyo, Japan. ${ }^{394}$ Sungkyunkwan University, Seoul, Republic of Korea. ${ }^{395}$ Finnish Institute of Occupational Health, Helsinki, Finland. ${ }^{396}$ St Vincent's Hospital, Sydney, New South Wales, Australia. ${ }^{397}$ University of New South Wales, Sydney, New South Wales, Australia. ${ }^{398}$ Karolinska Institutet, Stockholm, Sweden. ${ }^{399}$ Research Centre for Prevention and Health, Glostrup, Denmark. ${ }^{400}$ London School of Hygiene \& Tropical Medicine, London, UK. ${ }^{401}$ Diponegoro University, Semarang, Indonesia. ${ }^{402}$ University of Bari, Bari, Italy. ${ }^{403}$ University of Copenhagen, Copenhagen, Denmark. ${ }^{404}$ Institut Régional de Santé Publique, Ouidah, Benin. ${ }^{405}$ University of Bordeaux, Bordeaux, France. ${ }^{406} \mathrm{lzmir}$ Katip Çelebi University, Izmir, Turkey. ${ }^{407}$ University of Leuven, Leuven, Belgium. ${ }^{408}$ Institut National de la Santé et de la Recherche Médicale, Nancy, France. ${ }^{409}$ Bonn University, Bonn, Germany. ${ }^{410}$ Croatian Institute of Public Health, Zagreb, Croatia. ${ }^{411}$ National Institute of Public Health-National Institute of Hygiene, Warsaw, Poland. ${ }^{412}$ National Institute of Hygiene, Epidemiology and Microbiology, Havana, Cuba. ${ }^{413} \mathrm{Fu}$ Jen Catholic University, Taipei, Taiwan. ${ }^{414}$ National Statistic Office of Cabo Verde, Praia, Cabo Verde. ${ }^{415}$ Ministry of Health, Amman, Jordan. ${ }^{416}$ Central University of Kerala, 
Kasaragod, India. ${ }^{417} \mathrm{Health}$ Service of Murcia, Murcia, Spain. ${ }^{418}$ Institut d'Investigacio Sanitaria Illes Balears, Menorca, Spain. ${ }^{419}$ Universidad Centro-Occidental Lisandro Alvarado,

Barquisimeto, Venezuela. ${ }^{420}$ Dokuz Eylul University, Izmir, Turkey. ${ }^{421}$ University of Tampere Tays Eye Center, Tampere, Finland. ${ }^{422}$ Icahn School of Medicine at Mount Sinai, New York City, NY, USA. ${ }^{423}$ Utrecht University, Utrecht, The Netherlands. ${ }^{424}$ Hanoi University of Public Health, Hanoi, Vietnam. ${ }^{425}$ University Medical Center Utrecht, Utrecht, The Netherlands. ${ }^{426}$ Universitas Indonesia, Jakarta, Indonesia. ${ }^{427}$ Instituto de Investigación Sanitaria y Biomédica de Alicante, Alicante, Spain. ${ }^{428}$ North Karelian Center for Public Health, Joensuu, Finland. ${ }^{429}$ University of the Witwatersrand, Johannesburg, South Africa. ${ }^{430}$ Cork Institute of Technology, Cork, Ireland.

${ }^{431}$ Institute for Medical Research, Kuala Lumpur, Malaysia. ${ }^{432}$ Health Canada, Ottawa, Ontario,
Canada. ${ }^{433}$ Beijing Institute of Ophthalmology, Beijing, China. ${ }^{434}$ Xinjiang Medical University, Urumqi, China. ${ }^{435}$ Capital Medical University, Beijing, China. ${ }^{436}$ St George's, University of London, London, UK. ${ }^{437}$ Medical University of Vienna, Vienna, Austria. ${ }^{438}$ University of Oxford, Oxford, UK. ${ }^{439}$ Institute of Food and Nutrition Development of Ministry of Agriculture and Rural Affairs, Beijing, China. ${ }^{440}$ Children's Hospital of Fudan University, Shanghai, China. ${ }^{441}$ Penang Medical College, Penang, Malaysia. ${ }^{442}$ University of Cyprus, Nicosia, Cyprus. ${ }^{443}$ Iran University of Medical Sciences, Tehran, Iran. ${ }^{444}$ Jiangsu Provincial Center for Disease Control and Prevention, Nanjing, China. ${ }^{445}$ Sun Yat-sen University, Guangzhou, China. ${ }^{446}$ West Kazakhstan State Medical University, Aktobe, Kazakhstan. ${ }^{447}$ University of Ghana, Accra, Ghana. ${ }^{448}$ Deceased: Konrad

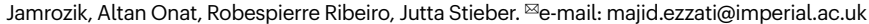




\section{Methods}

Our aim was to estimate trends in mean total, HDL and non-HDL cholesterol for 200 countries and territories (Supplementary Table 2). We used non-HDL cholesterol rather than low-density lipoprotein (LDL) cholesterol because most studies in our analysis had measured total cholesterol and HDL cholesterol, from which non-HDL cholesterol can be calculated through subtraction. By contrast, LDL cholesterol was directly measured in only around $14 \%$ of studies. When LDL cholesterol is not directly measured, its calculation requires data on triglycerides, which were available in approximately $64 \%$ of the studies. Furthermore, the most-commonly used estimation method-that is, the Friedewald equation-can be inaccurate, particularly at high levels of triglycerides $^{34}$. Non-HDL and LDL cholesterol were highly correlated (Pearson correlation coefficient $=0.94)$ in studies with data on both variables (Extended Data Fig. 9), because LDL cholesterol constitutes most of non-HDL cholesterol. Furthermore, non-HDL cholesterol predicts IHD risk at least as well as LDL cholesterol ${ }^{5,35}$, and can be measured at a lower cost than LDL cholesterol, which is relevant for how widely it can be used in low- and middle-income countries. Although non-HDL cholesterol is now commonly used in clinical guidelines ${ }^{36-38}$, LDL cholesterol continues to be a key target for treatment ${ }^{36,37}$, possibly because the interpretation of non-HDL cholesterol is more complex than LDL cholesterol alone. Specifically, an increase in non-HDL cholesterol could be due to the increase in LDL cholesterol or very-low-density

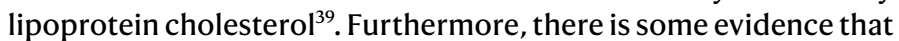
triglyceride levels are high in Asian populations, compared to levels seen in high-income western countries ${ }^{40}$. Therefore, data on non-HDL cholesterol can motivate dietary interventions to both reduce LDL cholesterol (for example, reducing saturated and trans fat intake) and triglyceride levels (for example, reducing refined carbohydrates and increasing omega-3 fatty acids) as well as treatments that lower LDL cholesterol (statins), alongside those that lower triglycerides (for example, fibrates).

\section{Data sources}

We used a database of population-based data on cardiometabolic risk factors collated by the NCD Risk Factor Collaboration (NCD-RisC), a worldwide network of health researchers and practitioners that systematically monitors the worldwide trends and variations in non-communicable disease (NCD) risk factors. The database was collated through multiple routes for identifying and accessing data. We accessed publicly available population-based multi-country and national measurement surveys (for example, Demographic and Health Surveys and surveys identified through the Inter-University Consortium for Political and Social Research and European Health Interview \& Health Examination Surveys Database). We requested, via the World Health Organization (WHO) and its regional and country offices, from ministries of health and other national health and statistical agencies to identify and access population-based surveys. Requests were also sent via the World Heart Federation to its national partners. We made a similar request to the co-authors of an earlier pooled analysis of cardiometabolic risk factors ${ }^{7,41-43}$, and invited the co-authors of the analysis to reanalyse data from their studies and join NCD-RisC. Finally, to identify major sources that were not accessed through the above routes, we searched and reviewed published studies as described in the Supplementary Information and invited all eligible studies to join NCD-RisC.

For each data source, we recorded the available information about the study population, start year and duration of measurement, sampling approach and measurement methods. The information about study population was used to establish that each data source was population-based, and to assess whether it covered the whole country, multiple subnational regions or one or a small number of communities, and whether it was rural, urban or combined.
We carefully checked all data sources in terms of how they met our inclusion and exclusion criteria listed below. We identified duplicate data sources by comparing studies from the same country and year. Additionally, all NCD-RisC members are asked periodically to review the list of sources from their country, to suggest additional sources not in the database, and to verify that the included data meet the inclusion criteria listed below and are not duplicates. The NCD-RisC database is continuously updated through the above routes and through regular contact with NCD-RisC members.

Anonymized individual record data from sources included in NCD-RisC were reanalysed according to a common protocol. Within each survey, we included participants aged 18 years and older who were not pregnant. We removed participants with implausible total cholesterol levels (defined as total cholesterol levels of $<1.75 \mathrm{mmoll}^{-1}$ or $>20 \mathrm{mmol}^{-1}$, or total cholesterol values that were lower than HDL cholesterol values) $(<0.05 \%$ of all participants with total cholesterol measurements) or HDL cholesterol levels (defined as HDL cholesterol levels of $<0.4 \mathrm{mmol} \mathrm{l}^{-1}$ or $>5 \mathrm{mmoll}^{-1}$, or total cholesterol values that were lower than HDL cholesterol values) $(<0.15 \%$ of all participants with HDL cholesterol measurements). When data on LDL cholesterol were also available, we removed individuals for whom the sum of LDL and HDL cholesterol level surpassed total cholesterol level by more than is plausible based on the limits to errors in their measurement (following the CDC Cholesterol Reference Method Laboratory Network (CRMLN) standards, these errors were set at $8.9 \%$ for total cholesterol, $13 \%$ for HDL cholesterol and $12 \%$ for LDL cholesterol) $(<0.06 \%$ of all participants with total cholesterol and HDL cholesterol measurements $)^{44-46}$.

We calculated mean total cholesterol, mean HDL cholesterol and mean non-HDL cholesterol, and associated standard errors and sample sizes, by sex and age group (18-19 years, 20-29 years, followed by 10 -year age groups and $80+$ years). All analyses incorporated appropriate sample weights and complex survey design in calculating age-sex-specific means when applicable. To ensure summaries were prepared according to the study protocol, computer code was provided to NCD-RisC members who requested assistance. All submitted data were checked independently by at least two researchers. Questions and clarifications were discussed with NCD-RisC members and resolved before the data were incorporated in the database.

Finally, we obtained data not accessed through the above routes by extracting data from published reports of all additional national health surveys identified through the above-described strategies, as well as eight sites of the WHO Multinational MONItoring of trends and determinants in CArdiovascular disease (MONICA) project that were not deposited in the MONICA Data Centre. Data were extracted from published reports only when reported by sex and in age groups no wider than 20 years. We also used data from a previous pooling study ${ }^{7}$ when such data did not overlap with those accessed through the above routes.

\section{Data inclusion and exclusion}

Data sources were included in NCD-RisC database if: (1) measured data on total, LDL, HDL cholesterol and/or triglycerides were available; (2) study participants were 10 years of age or older; (3) data were collected using a probabilistic sampling method with a defined sampling frame; (4) data were from population samples at the national, subnational (covering one or more subnational regions, more than three urban communities or more than five rural communities) or community (one or a small number of communities) level; (5) data were collected in or after 1950; and (6) data were from the countries and territories listed in Supplementary Table 2.

We excluded all data sources that included only hypercholesterolaemia or dyslipidaemia diagnosis history or medication status without measurement of cholesterol levels. We also excluded data sources on population subgroups for which the lipid profile may differ systematically from the general population, including: (1) studies that had included or excluded people on the basis of their health status or 
cardiovascular risk; (2) studies for which the participants were only from ethnic minorities; (3) studies that had recruited only specific educational, occupational or socioeconomic subgroups, with the exception noted below; and (4) studies that had recruited participants through health facilities, with the exception noted below.

We used school-based data in countries and for age-sex groups, for which secondary school enrolment was $70 \%$ or higher. We used data for which the sampling frame was health insurance schemes in countries in which at least $80 \%$ of the population was insured. Finally, we used data collected through general practice and primary-care systems in high-income and central European countries with universal insurance, because contact with the primary-care systems tends to be as good as or better than response rates for population-based surveys. We used data sources regardless of fasting status, because the differences between fasting and non-fasting measurements are negligible for total, non-HDL and $\mathrm{HDL}$ cholesterol ${ }^{39}$, and therefore non-fasting lipid profiles are now widely endorsed for the estimation of cardiovascular risk ${ }^{36,37}$.

\section{Data used in the analysis}

For this paper, we used data from the NCD-RisC database for years 1980 to 2018 and individuals aged 18 years and older. A list of the data sources that we used in this analysis and their characteristics is provided in Supplementary Table 1. The data comprised 1,127 population-based measurement surveys and studies that included measurements of blood lipids on 102.6 million participants aged 18 years and older. We had at least one data source for 161 of the 200 countries that we made estimates for, covering $92.4 \%$ of the world's population in 2018 (Extended Data Fig. 1); and at least two data sources for 104 countries (87.5\% of the world population). Of these 1,127 sources, 409 (36.3\%) sampled from national populations, 250 (22.2\%) covered one or more subnational regions, and the remaining 468 (41.5\%) were from one or a small number of communities. Regionally, data availability ranged from around 2 data sources per country in sub-Saharan Africa to approximately 35 sources per country in the high-income Asia-Pacific region. In total, 454 data sources (40.3\%) were from years before 2000 and the remaining 673 (59.7\%) were collected from 2001 onwards.

\section{Adjusting for the differences in mean cholesterol between portable device and laboratory measurements}

In 112 (10\%) of the 1,127 data sources used in our analysis (11.5\% and 5.8\% of age-sex-specific data points for total and HDL cholesterol, respectively) lipids were measured using a portable device. Some portable devices have narrower analytical ranges than laboratory methods, which results in truncations of blood cholesterol data that are outside their range (Supplementary Table 3). This may in turn affect the population mean. Although cholesterol concentrations that fall outside the analytical range are displayed as 'high' (above the measurement range) or 'low' (below the measurement range) by these devices, different surveys record and code cholesterol concentrations outside the analytical range in different ways, for example using 'too low', 'too high' and 'error' codes; assigning the minimum or maximum value to individuals whose cholesterol was below or above the analytical range, respectively; setting values outside the analytical range to missing; and so on. We used an approach that treated surveys with such data consistently.

Specifically, we first dropped all participants with cholesterol levels below and at the minimum, and at and above the maximum, values of the analytical range of each portable device before calculating the mean cholesterol (Supplementary Table 3). We then developed conversion regressions to adjust the mean cholesterol levels measured using a portable device (calculated over the restricted range, Supplementary Table 3) to the levels expected using laboratory measurements. The dependent variable in each regression was mean total, non-HDL or HDL cholesterol for the full range, and the main independent variable was mean total, non-HDL or HDL cholesterol over the above-mentioned restricted cholesterol range of the portable devices. The regression coefficients were estimated from data sources for which lipids were measured in a laboratory, and thus had the full range of measurement and could be used to calculate both dependent and independent variables. When estimating the regression coefficients, we constructed the dependent variable using the full data, and the independent variable by dropping the values outside the above-mentioned restricted cholesterol range of each device, mimicking those that would be expected if a portable device had been used. Separate models were developed according to the specific range of the different portable devices. All regressions included terms for age and sex, as well as interactions between predictors and age and sex, based on the Bayesian information criterion $^{47}$. The regressions for mean non-HDL cholesterol also included mean total cholesterol and mean HDL cholesterol because non-HDL cholesterol is calculated from total cholesterol and HDL cholesterol. We excluded data points for which there were fewer than 25 individuals for the purpose of estimating the coefficients of these regressions. All sources of uncertainty in the conversion-including the sampling uncertainty of the original data, the uncertainty of the regression coefficients and residuals - were carried forward by using repeated draws from their respective distributions. The regression coefficients and number of data points used to estimate the coefficients are shown in Supplementary Table 4.

\section{Statistical analysis}

We used a statistical model to estimate mean total, non-HDL and HDL cholesterol by country, year, sex and age using all of the available data. The model is described in detail in a statistical paper and related substantive papers ${ }^{8,32,33,48}$; the computer code is available at http://www. ncdrisc.org/. In summary, we organized countries into 21 regions, mainly based on geography and national income; these regions were further aggregated into 9 'super-regions' (Supplementary Table 2). The model had a hierarchical structure in which estimates for each country and year were informed by its own data, if available, and by data from other years in the same country and from other countries, especially countries in the same region or super-region with data for similar time periods. The extent to which estimates for each country-year are influenced by data from other years and other countries depends on whether the country has data, the sample size of data, whether or not they are national, and the within-country and within-region data variability. The model incorporated nonlinear time trends comprising linear terms and a second-order random walk. The age association of blood lipids was modelled using a cubic spline to allow nonlinear age patterns, which might vary across countries. The model accounted for the possibility that blood lipids in subnational and community samples might systematically differ from nationally representative ones; and/ or have larger variation. These features were implemented by including data-driven fixed-effect and random-effect terms for subnational and community data. The fixed effects adjust for systematic differences between subnational or community studies and national studies. The random effects allow national data to have larger influence on the estimates than subnational or community data with similar sample sizes. The model also accounted for rural-urban differences in blood lipids, through the use of data-driven fixed effects for rural-only and urban-only studies. These rural and urban effects were weighted by the difference between study-level and country-level urbanization in the year in which the study was done. The proportion of the national population living in urban areas was also included as a predictor (covariate) in the model. The model for mean non-HDL and HDL cholesterol also used age-standardized mean total cholesterol as a covariate.

We fitted the statistical model with the Markov chain Monte Carlo (MCMC) algorithm, and obtained 5,000 post-burn-in samples from the posterior distribution of model parameters, which were in turn used to obtain the posterior distributions of mean total, non-HDL and HDL cholesterol. We calculated average change in mean total, HDL and non-HDL cholesterol across the 39 years of analysis (reported as change 
per decade). Age-standardized estimates were generated by taking weighted averages of age-sex-specific estimates, using the WHO standard population. Estimates for regions and the world were calculated as population-weighted averages of the constituent country estimates by age group and sex. The reported credible intervals represent the 2.5-97.5th percentiles of the posterior distributions. We also report the posterior probability that an estimated increase or decrease represents a truly increasing or decreasing trend as opposed to a chance observation. We performed all analyses by sex, because blood lipids levels and trends are different in men and women.

\section{Validation of statistical model}

We tested how well our statistical model predicts missing data, known as external predictive validity, in two different tests. In the first test, we held out all data from $10 \%$ of countries with data (that is, created the appearance of countries with no data where we actually had data). The countries for which the data were withheld were randomly selected from the following three groups: data rich ( 5 or more data sources, with at least one data source after the year 2000), data poor (1 data source) and average data availability ( $2-4$ data sources). In the second test, we assessed other patterns of missing data by holding out $10 \%$ of our data sources, again from a mix of data-rich, data-poor and average-data countries, as defined above. For a given country, we either held out a random half of the data of a country or all of the 2000-2018 data of the country to determine, respectively, how well we filled in the gaps for countries with intermittent data and how well we estimated in countries without recent data. In both tests, we then fitted the model to the remaining $90 \%$ of the countries (test 1 ) or data sources (test 2 ) and made estimates of the held-out observations. We repeated each test five times, holding out a different subset of data in each repetition. In both tests, we calculated the differences between the held-out data and the estimates. We also calculated the $95 \%$ credible intervals of the estimates; in a model with good external predictive validity, $95 \%$ of held-out values would be included in the $95 \%$ credible intervals.

Our statistical model performed well in the external validation tests, that is, in estimating mean cholesterol when data were missing. The estimates of mean total, non-HDL and HDL cholesterol were unbiased, as evidenced with median errors that were very close to zero globally for every outcome and test, and less than $\pm 0.30 \mathrm{mmoll}^{-1}$ in every subset of withheld data except for women in the high-income Asia-Pacific region in test 1 for non-HDL cholesterol (median error $0.47 \mathrm{mmoll}^{-1}$ ) and men in south Asia in test 2 for non-HDL cholesterol (median error $-0.33 \mathrm{mmol} \mathrm{I}^{-1}$ ) (Supplementary Table 5). The $95 \%$ credible intervals of estimated means covered $83-92 \%$ and $75-83 \%$ of true data globally in the first and second tests, respectively. In subsets, coverage ranged from $47 \%$ to $100 \%$, but was mostly greater than $75 \%$, with coverage generally lower in test 2 than test 1 . Median absolute errors ranged from 0.07 to $0.23 \mathrm{mmol}^{-1}$ globally for different outcomes and sexes, and were no more than $0.45 \mathrm{mmol}^{-1}$ in all subsets of withheld data, except for women in the high-income Asia-Pacific region for non-HDL cholesterol in test 1 (median absolute error $0.47 \mathrm{mmoll}^{-1}$ ).

\section{Calculation of the number of deaths attributable to high cholesterol}

We estimated the number of deaths from IHD and ischaemic stroke attributable to high non-HDL cholesterol. For each country, year, sex and age group, we first calculated the population attributable fractions-that is, the proportion of deaths from IHD and ischaemic stroke that would have been prevented if non-HDL cholesterol levels were at an optimal level (defined as a mean of 1.8-2.2 $\mathrm{mmoll}^{-1}$ ) in the population $^{6,49}$. For these calculations, we used age-specific relative risks from meta-analyses of prospective cohort studies ${ }^{4,5,50}$. The number of IHD and ischaemic stroke deaths attributable to high non-HDL cholesterol was calculated for each country-year-age-sex group by multiplying the cause-specific population attributable fractions by the cause-specific deaths from the Global Burden of Disease study in 1990 and 2017 (the earliest and latest years with cause-specific mortality data).

\section{Strengths and limitations}

The strengths of our study include its scope in making consistent and comparable estimates of trends in blood cholesterol and its cardiovascular disease mortality burden, over almost four decades for all of the countries in the world, including global estimates of non-HDL and HDL cholesterol. We used a large amount of population-based data, which came from countries in which $92 \%$ of the global adult population lives. We used only data from studies that had measured blood lipids to avoid bias in self-reported data. Data were analysed according to a consistent protocol, and the characteristics and quality of data from each country were rigorously verified through repeated checks by NCD-RisC members. We pooled data using a statistical model that took into account the epidemiological features of cholesterol, including nonlinear time trends and age associations. Our statistical model used all available data while giving more weight to national data than to subnational and community sources.

Similar to all global analyses, our study is affected by some limitations. Despite our extensive efforts to identify and access worldwide population-based data, some countries had no or few data sources, especially those in sub-Saharan Africa, the Caribbean, central Asia and Melanesia. Estimates for these countries relied mostly or entirely on the statistical model, which shares information across countries and regions through its hierarchy. Data scarcity is reflected in wider uncertainty intervals of our estimates for these countries and regions, highlighting the need for national NCD-oriented surveillance. The distribution of lipids measured in a population using a portable device, which was used in $10 \%$ of our studies, may be truncated and may therefore affect the population mean. To overcome this issue, we developed conversion regressions to adjust mean cholesterol levels measured using a portable device to the levels expected in laboratory measurements; the conversion regressions used for this purpose had good predictive accuracy. Although most studies had measured cholesterol in serum samples, around $7 \%$ had used plasma samples. As cholesterol measured in plasma and serum samples differ ${ }^{51}$ by only about $3 \%$, adjusting for plasma-serum differences would have little effect on our results, as seen in a previous analysis ${ }^{14}$. Although methods to measure total and HDL cholesterol have evolved over time, since the 1950s there have been systematic efforts to standardize lipid measurements that have resulted in increased comparability between different methods. In our analysis, $90 \%$ of studies measured lipids in a laboratory; of these studies more than $60 \%$ for total cholesterol and more than $70 \%$ for HDL cholesterol participated in a lipid standardization programme or quality control scheme. We did not analyse emerging lipid markers such as apolipoprotein B and apolipoprotein A-I, because they are neither commonly measured in population-based health surveys, nor routinely used in clinical practice ${ }^{36}$.

\section{Comparison with other studies}

There are no global analyses on trends in lipid fractions for comparison with our results. Our findings for total cholesterol were largely consistent with the only other previous analysis ${ }^{7}$, but we estimated a larger decrease in mean total cholesterol in high-income western countries and central Europe, and a larger increase in southeast Asia, because we had an additional decade of data compared with the earlier global analysis. Therefore, although the highest mean total cholesterol levels reported previously ${ }^{7}$, for 2008 , were still in high-income western countries, we estimated that in 2018 total cholesterol was equally high or higher in southeast Asia. Our findings on mean total cholesterol trends are also largely consistent with previous multi- and single-country reports $^{14,15,17-21,52-73}$. Differences from previous studies-for example, in Italy ${ }^{61}$, Lithuania $^{63}$, the Netherlands ${ }^{65}$, Russian Federation ${ }^{69}$ and in some countries that participated in the MONICA Project ${ }^{52}-$ mostly arise 
because our study covered a longer period and used a larger number of data sources. Studies ${ }^{15,18,54,63,66,70,74-77}$ that have reported trends in lipid fractions for a period longer than 15 years have found changes in non-HDL cholesterol (or in LDL cholesterol for some studies) that were consistent with our results.

\section{Reporting summary}

Further information on research design is available in the Nature Research Reporting Summary linked to this paper.

\section{Data availability}

Estimates of mean total, non-HDL and HDL cholesterol by country, year and sex are available at http://www.ncdrisc.org/. Input data from publicly available sources can also be downloaded from http://www. ncdrisc.org/. For other data sources, contact information for data providers can be obtained from http://www.ncdrisc.org/.

\section{Code availability}

The computer code for the Bayesian hierarchical model used in this work is available at http://www.ncdrisc.org/.

34. Martin, S. S. et al. Friedewald-estimated versus directly measured low-density lipoprotein cholesterol and treatment implications. J. Am. Coll. Cardiol. 62, 732-739 (2013).

35. Cui, Y. et al. Non-high-density lipoprotein cholesterol level as a predictor of cardiovascular disease mortality. Arch. Intern. Med. 161, 1413-1419 (2001).

36. Grundy, S. M. et al. 2018 AHA/ACC/AACVPR/AAPA/ABC/ACPM/ADA/AGS/APhA/ASPC/ NLA/PCNA guideline on the management of blood cholesterol: a report of the American College of Cardiology/American Heart Association Task Force on clinical practice guidelines. Circulation 139, e1082-e1143 (2019).

37. Mach, F. et al. $2019 \mathrm{ESC} / \mathrm{EAS}$ guidelines for the management of dyslipidaemias: lipid modification to reduce cardiovascular risk. Eur. Heart J. 41, 111-188 (2020).

38. Expert Dyslipidemia Panel of the International Atherosclerosis Society. An International Atherosclerosis Society Position Paper: global recommendations for the management of dyslipidemia-full report. J. Clin. Lipidol. 8, 29-60 (2014).

39. Nordestgaard, B. G. et al. Fasting is not routinely required for determination of a lipid profile: clinical and laboratory implications including flagging at desirable concentration cut-points-a joint consensus statement from the European Atherosclerosis Society and European Federation of Clinical Chemistry and Laboratory Medicine. Eur. Heart J. 37, 1944-1958 (2016).

40. Bilen, O., Kamal, A. \& Virani, S. S. Lipoprotein abnormalities in South Asians and its association with cardiovascular disease: current state and future directions. World J. Cardiol. 8, 247-257 (2016)

41. Danaei, G. et al. National, regional, and global trends in systolic blood pressure since 1980: systematic analysis of health examination surveys and epidemiological studies with 786 country-years and 5.4 million participants. Lancet 377, 568-577 (2011).

42. Danaei, G. et al. National, regional, and global trends in fasting plasma glucose and diabetes prevalence since 1980: systematic analysis of health examination surveys and epidemiological studies with 370 country-years and 2.7 million participants. Lancet 378 31-40 (2011)

43. Finucane M. M et al National, regional and global trends in body-mass index since 1980: systematic analysis of health examination surveys and epidemiological studies with 960 country-years and 9.1 million participants. Lancet 377, 557-567 (2011).

44. Cholesterol Reference Method Laboratory Network. Total Cholesterol Certification Protocol for Manufacturers. https://www.cdc.gov/labstandards/pdf/crmln/ RevisedTCprotocolOct04.pdf (Cholesterol Reference Method Laboratory Network, 2004).

45. Cholesterol Reference Method Laboratory Network. HDL Cholesterol Certification Protocol for Manufacturers. https://www.cdc.gov/labstandards/pdf/crmln/HDL Certification_Protocol-508.pdf (Cholesterol Reference Method Laboratory Network, 2018).

46. Cholesterol Reference Method Laboratory Network. LDL Cholesterol Certification Protocol for Manufacturers. https://www.cdc.gov/labstandards/pdf/crmln/LDL Certification_Protocol-508.pdf (Cholesterol Reference Method Laboratory Network, 2018).

47. Schwarz, G. Estimating the dimension of a model. Ann. Stat. 6, 461-464 (1978).

48. Finucane, M. M. Paciorek, C. J. Danaei, G. \& Ezzati, M. Bayesian estimation of population-level trends in measures of health status. Stat. Sci. 29, 18-25 (2014).

49. Ezzati, M., Lopez, A. D., Rodgers, A., Vander Hoorn, S. \& Murray, C. J. Selected major risk factors and global and regional burden of disease. Lancet 360, 1347-1360 (2002).

50. Singh, G. M. et al. The age-specific quantitative effects of metabolic risk factors on cardiovascular diseases and diabetes: a pooled analysis. PLoS ONE 8, e65174 (2013).

51. National Cholesterol Education Program. Recommendations on Lipoprotein Measurement. From the Working Group on Lipoprotein Measurement. NIH Publication No. 95-3044 (National Institutes of Health, National Heart, Lung, and Blood Institute, 1995).

52. Evans, A. et al. Trends in coronary risk factors in the WHO MONICA project. Int. J. Epidemiol. 30, S35-S40 (2001).

53. Bennett, S. A. \& Magnus, P. Trends in cardiovascular risk factors in Australia. Results from the National Heart Foundation's Risk Factor Prevalence Study, 1980-1989. Med. J. Aust. 161, 519-527 (1994)
54. Cífková, R. et al. Longitudinal trends in major cardiovascular risk factors in the Czech population between 1985 and 2007/8. Czech MONICA and Czech post-MONICA. Atherosclerosis 211, 676-681 (2010).

55. Sun, J. Y. et al. The changing trend of serum total cholesterol in Beijing population aged 25 - 64 years during 1984 - 1999 (article in Chinese). Zhonghua Nei Ke Za Zhi 45, 980-984 (2006).

56. Afzal, S., Tybjærg-Hansen, A., Jensen, G. B. \& Nordestgaard, B. G. Change in body mass index associated with lowest mortality in Denmark, 1976-2013. J. Am. Med. Assoc. 315, 1989-1996 (2016).

57. Ferrières, J. et al. Trends in plasma lipids, lipoproteins and dyslipidaemias in French adults, 1996-2007. Arch. Cardiovasc. Dis. 102, 293-301 (2009).

58. Truthmann, J. et al. Changes in mean serum lipids among adults in Germany: results from National Health Surveys 1997-99 and 2008-11. BMC Public Health 16, 240 (2016).

59. Sigfusson, $\mathrm{N}$. et al. Decline in ischaemic heart disease in lceland and change in risk factor levels. Br. Med. J. 302, 1371-1375 (1991)

60. Gupta, R. et al. Twenty-year trends in cardiovascular risk factors in India and influence of educational status. Eur. J. Prev. Cardiol. 19, 1258-1271 (2012).

61. Giampaoli, S. et al. Cardiovascular health in Italy. Ten-year surveillance of cardiovascular diseases and risk factors: Osservatorio Epidemiologico Cardiovascolare/Health Examination Survey 1998-2012. Eur. J. Prev. Cardiol. 22, 9-37 (2015).

62. Iso, $\mathrm{H}$. Changes in coronary heart disease risk among Japanese. Circulation 118 2725-2729 (2008).

63. Luksiene, D. et al. Trends in prevalence of dyslipidaemias and the risk of mortality in Lithuanian urban population aged $45-64$ in relation to the presence of the dyslipidaemias and the other cardiovascular risk factors. PLoS ONE 9, e100158 (2014).

64. Uusitalo, U. et al. Fall in total cholesterol concentration over five years in association with changes in fatty acid composition of cooking oil in Mauritius: cross sectional survey. Br. Med. J. 313, 1044-1046 (1996).

65. Koopman, C. et al. Trends in risk factors for coronary heart disease in the Netherlands BMC Public Health 16, 835 (2016).

66. Metcalf, P. et al. Trends in major cardiovascular risk factors in Auckland, New Zealand: 1982 to 2002-2003. N. Z. Med. J. 119, U2308 (2006).

67. Sharashova, E., Wilsgaard, T. \& Brenn, T. Resting heart rate on the decline: the Troms $\varnothing$ Study 1986-2007. Int. J. Epidemiol. 44, 1007-1017 (2015).

68. Pajak, A et al. Changes over time in blood lipids and their correlates in Polish rural and urban populations: the Poland-United States Collaborative Study in cardiopulmonary disease epidemiology. Ann. Epidemiol. 7, 115-124 (1997)

69. Vlasoff, T. et al. Ten year trends in chronic disease risk factors in the Republic of Karelia, Russia. Eur. J. Public Health 18, 666-673 (2008).

70. Bovet, P. et al. Divergent fifteen-year trends in traditional and cardiometabolic risk factors of cardiovascular diseases in the Seychelles. Cardiovasc. Diabetol. 8, 34 (2009).

71. Serra-Majem, L. et al. Trends in blood lipids and fat soluble vitamins in Catalonia, Spain (1992-2003). Public Health Nutr. 10, 1379-1388 (2007).

72. Berg, C. M. et al. Trends in blood lipid levels, blood pressure, alcohol and smoking habits from 1985 to 2002: results from INTERGENE and GOT-MONICA. Eur. J. Cardiovasc. Prev. Rehabil. 12, 115-125 (2005).

73. Wietlisbach, V., Paccaud, F., Rickenbach, M. \& Gutzwiller, F. Trends in cardiovascular risk factors (1984-1993) in a Swiss region: results of three population surveys. Prev. Med. 26, 523-533 (1997).

74. Leiviskä, J. et al. What have we learnt about high-density lipoprotein cholesterol measurements during 32 years? Experiences in Finland 1980-2012. Clin. Chim. Acta $\mathbf{4 1 5}$ 118-123 (2013).

75. Yokoyama, S. et al. High-density lipoprotein levels have markedly increased over the past twenty years in Japan. J. Atheroscler. Thromb. 21, 151-160 (2014).

76. Hardoon, S. L. et al. Assessing the impact of medication use on trends in major coronary risk factors in older British men: a cohort study. Eur. J. Cardiovasc. Prev. Rehabil. 17 502-508 (2010).

77. Carroll, M. D. et al. Trends in serum lipids and lipoproteins of adults, 1960-2002. J. Am. Med. Assoc. 294, 1773-1781 (2005).

Acknowledgements This study was funded by a Wellcome Trust (Biomedical Resource \& Multi-User Equipment grant 01506/Z/13/Z) and the British Heart Foundation (Centre of Research Excellence grant RE/18/4/34215). C.T. was supported by a Wellcome Trust Research Training Fellowship (203616/Z/16/Z). The authors alone are responsible for the views expressed in this Article and they do not necessarily represent the views, decisions, or policies of the institutions with which they are affiliated.

Author contributions M.E. and G.D. designed the study and oversaw research. C.T., B.Z., H.B. and R.C.L. led the data collection. The other authors contributed to study design; and collected, reanalysed, checked and pooled data. C.T. analysed pooled data and prepared results. C. . E.G. and M.E. wrote the first draft of the manuscript with input from the other authors.

Competing interests M.E. reports a charitable grant from the AstraZeneca Young Health Programme, and personal fees from Prudential, Scor and Third Bridge, outside the submitted work. The other authors declare no competing interests.

Additional information

Supplementary information is available for this paper at https://doi.org/10.1038/s41586-020 2338-1.

Correspondence and requests for materials should be addressed to M.E.

Peer review information Nature thanks Frank Hu and Pekka Jousilahti for their contribution to the peer review of this work.

Reprints and permissions information is available at http://www.nature.com/reprints 


\section{Article}
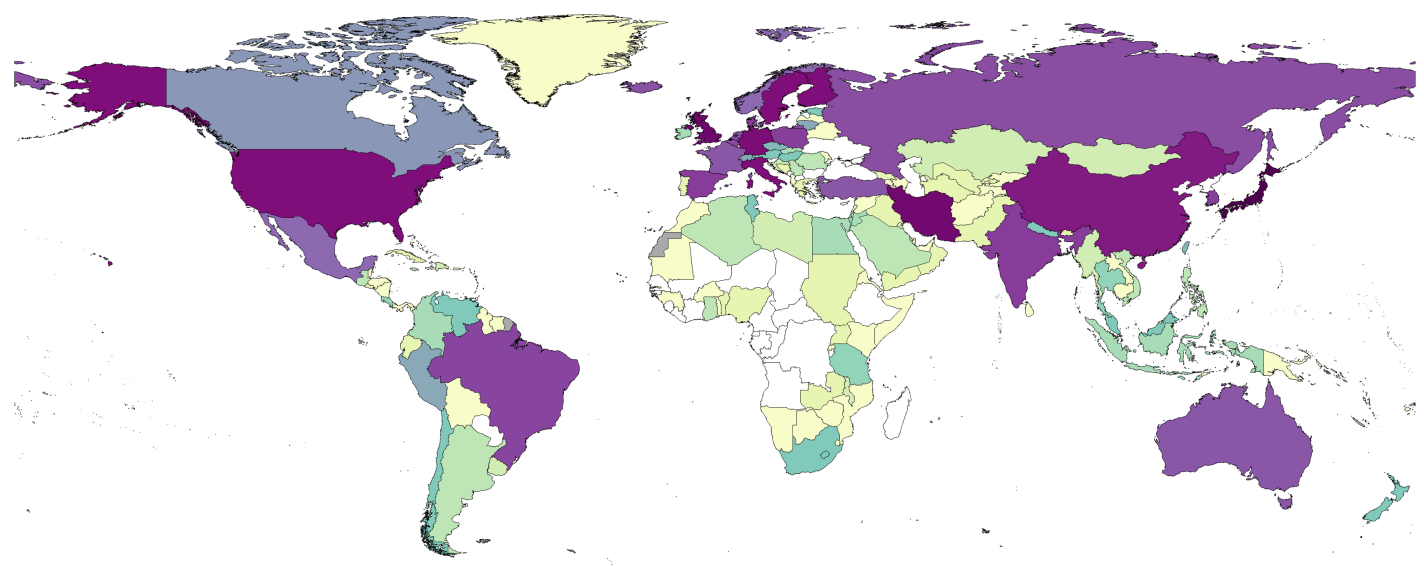

\section{Number of sources in each country}

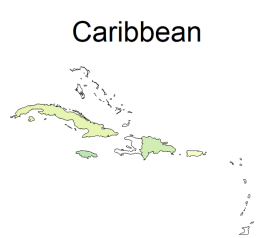

$$
\begin{aligned}
& \square \text { American Samoa } \\
& \square \text { Bahrain } \\
& \square \text { Bermuda } \\
& \square \text { Brunei Darussalam } \\
& \square \text { Cabo Verde } \\
& \square \text { Comoros } \\
& \square \text { Cook Islands }
\end{aligned}
$$

$\begin{array}{ll}\square \text { Fiji } & \square \text { Montenegro } \\ \square \text { French Polynesia } & \square \text { Nauru } \\ \square \text { Kiribati } & \square \text { Niue } \\ \square \text { Maldives } & \square \text { Palau } \\ \square \text { Marshall Islands } & \square \text { Samoa } \\ \square \text { Mauritius } & \square \text { Sao Tome and Principe } \\ \square \text { Federated States of Micronesia } & \end{array}$

$\square$ Seychelles
$\square$ Solomon Islands
$\square$ Tokelau
$\square$ Tonga
$\square$ Tuvalu
$\square$ Vanuatu

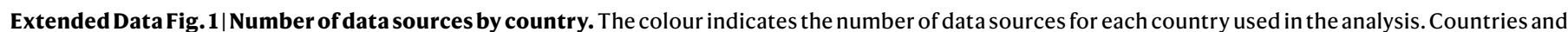
territories that were not included in the analysis are coloured in grey. 


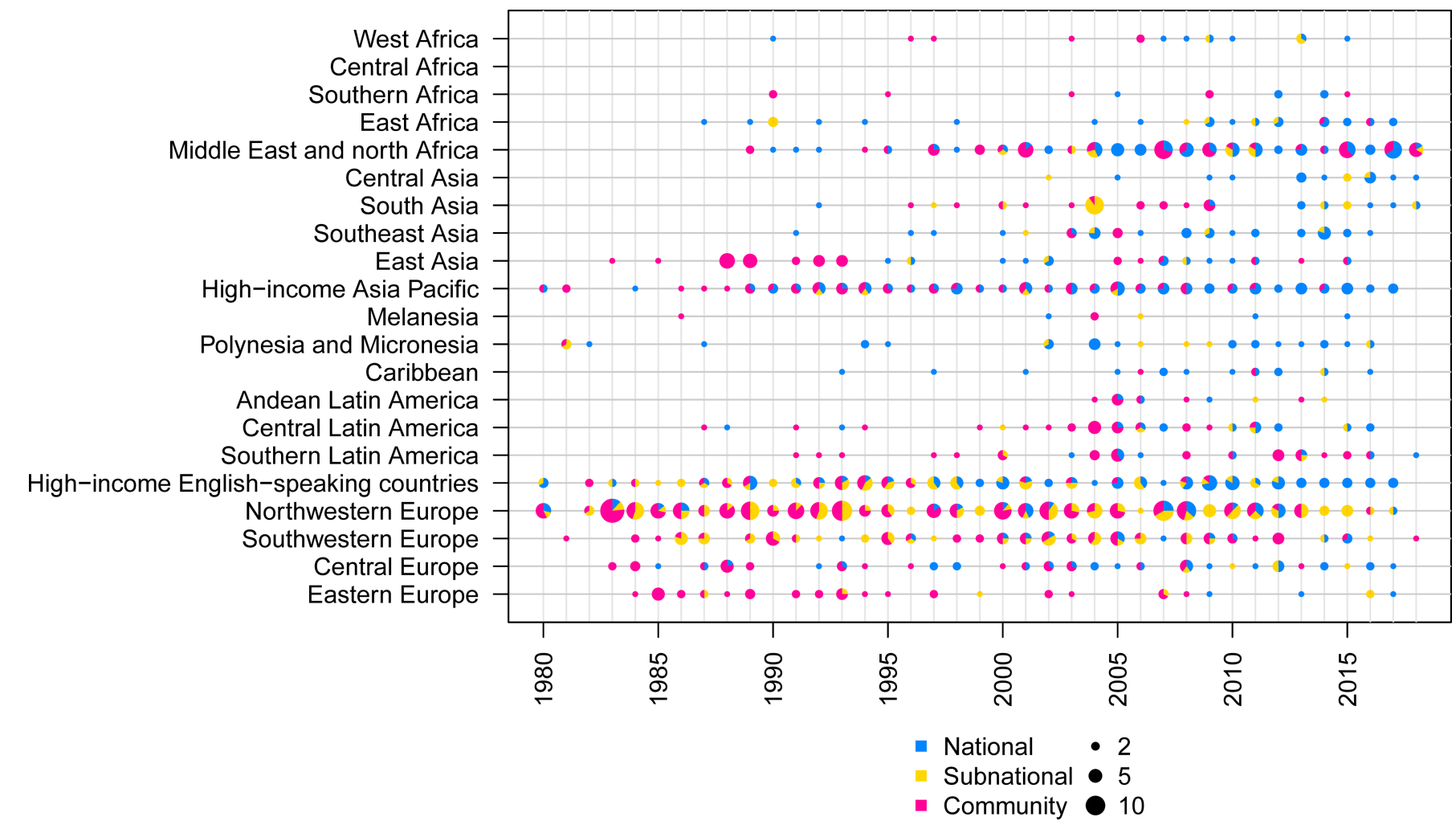

Extended Data Fig. 2 | Number of data sources by region and year. The size of each circle shows the number of data sources for each region and year, and the colours indicate the relative size of national, subnational and community data sources. 


\section{Women}

High-income Asia Pacific Northwestern Europe High-income English-speaking countries Southwestern Europe

Central Europe

Eastern Europe

East Asia

Southern Africa

Southern Latin America

Caribbean

World

Central Asia

Southeast Asia

Middle East and north Africa

Central Africa

Andean Latin America

West Africa

East Africa

Central Latin America

South Asia

Melanesia

Polynesia and Micronesia

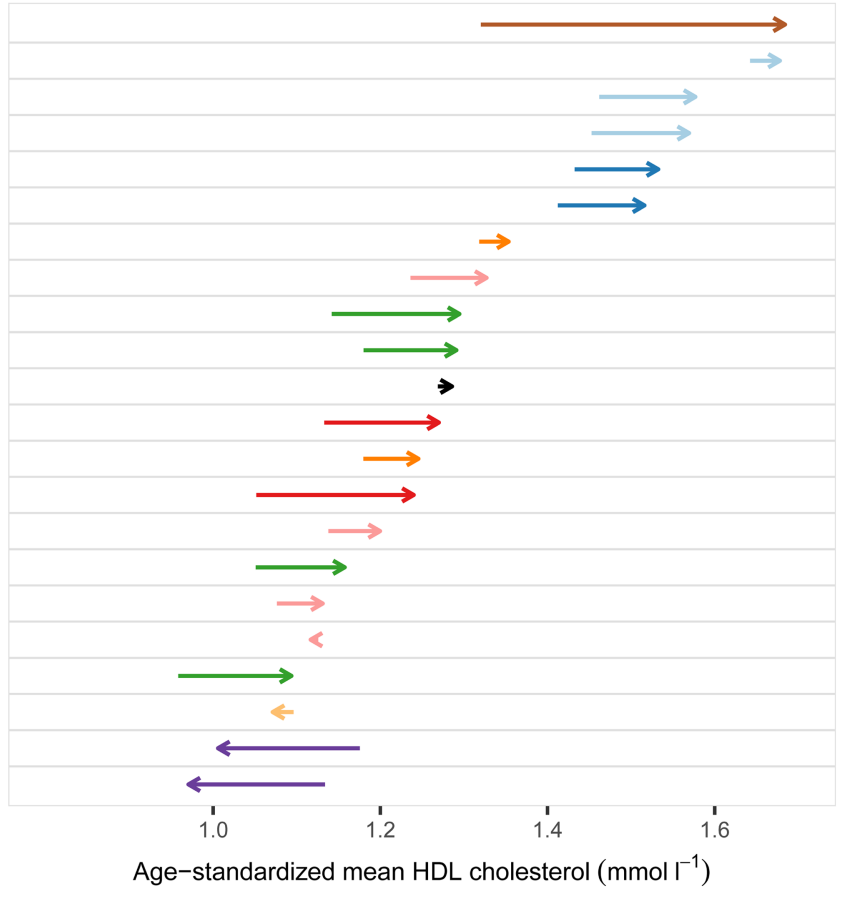

Men

High-income Asia Pacific Eastern Europe

Northwestern Europe

High-income English-speaking countries Central Europe Southwestern Europe Southern Africa

East Asia

Caribbean

World

Central Asia

Southern Latin America

Southeast Asia

Middle East and north Africa

Central Africa

Andean Latin America

West Africa

East Africa

South Asia

Central Latin America

Melanesia

Polynesia and Micronesia

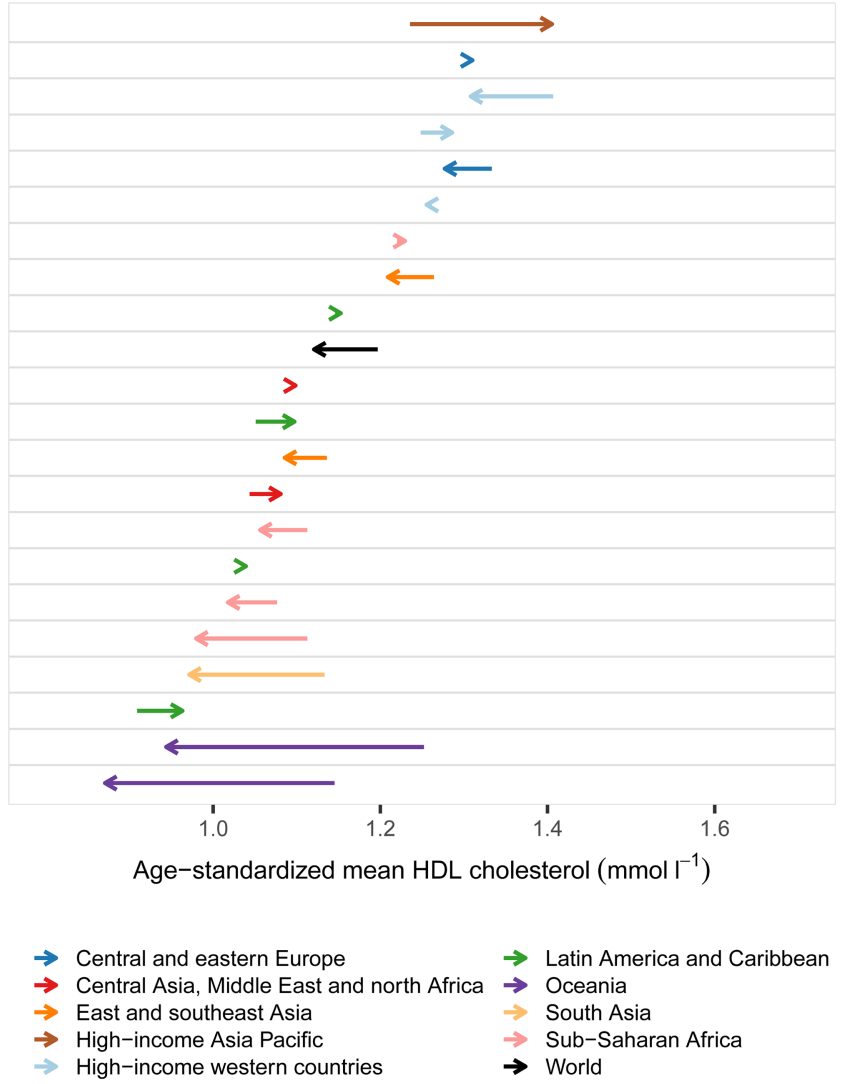

Extended Data Fig. 3 | Change in age-standardized mean HDL cholesterol between 1980 and 2018 by region for women and men. The start of the arrow shows the level in 1980 and the head shows the level in 2018 . One $\mathrm{mmoll}^{-1}$ is equivalent to $38.61 \mathrm{mg} \mathrm{dl}^{-1}$. 


\section{Women}

Northwestern Europe

Eastern Europe

High-income English-speaking countries

Southwestern Europe

Central Europe

Southern Latin America

High-income Asia Pacific

Central Latin America

Central Asia

Southern Africa

Andean Latin America

Middle East and north Africa

Caribbean

Polynesia and Micronesia

South Asia

Central Africa

Melanesia

East Asia

West Africa

East Africa

Southeast Asia

$-1.0$

$-0.5$

Change in age-standardized mean cholesterol $\left(\mathrm{mmol} \mathrm{I}^{-1}\right)$

Men

Northwestern Europe

Southwestern Europe

High-income English-speaking countries

Eastern Europe

Central Europe

Southern Latin America

Central Latin America

Southern Africa

Andean Latin America

Polynesia and Micronesia

Middle East and north Africa

Central Asia

Caribbean

High-income Asia Pacific

South Asia

Melanesia

Central Africa

East Africa

West Africa

East Asia

Southeast Asia
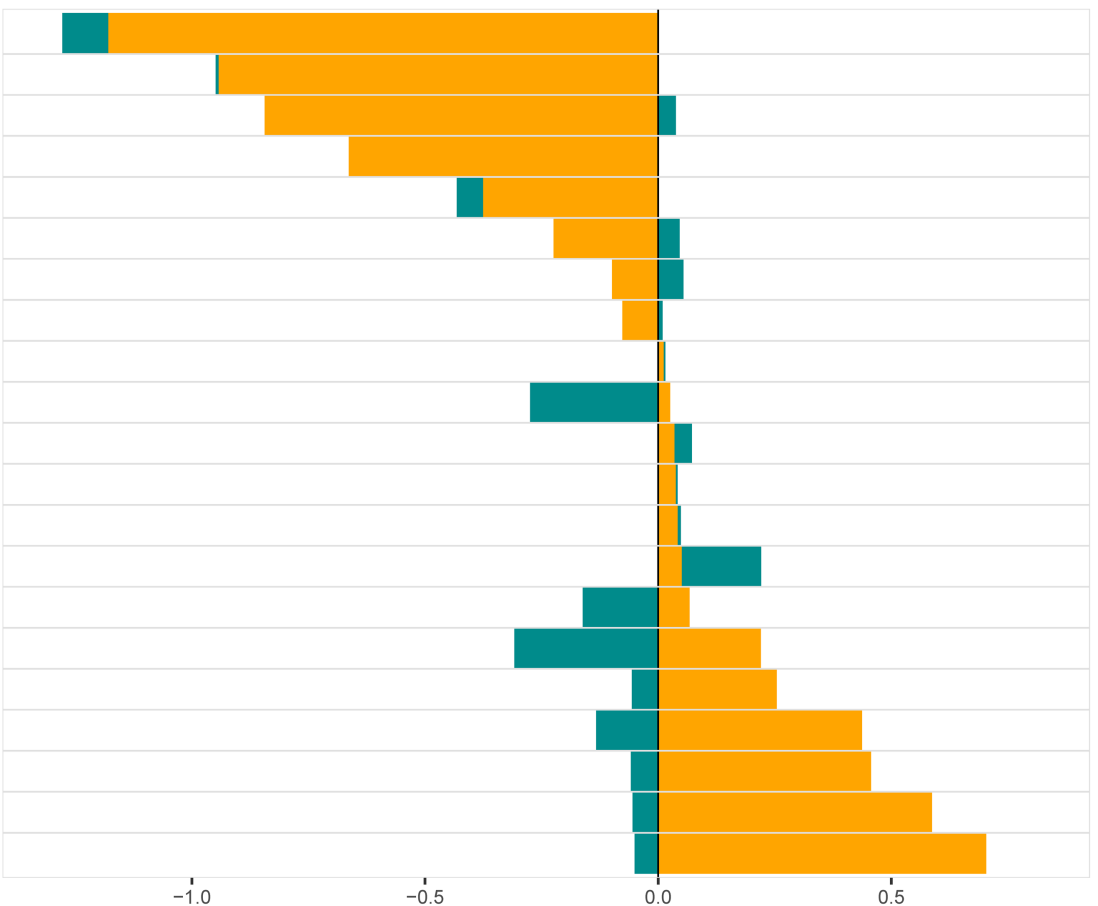

Change in age-standardized mean cholesterol $\left(\mathrm{mmol} \mathrm{I}^{-1}\right)$

Change in age-standardized mean non-HDL cholesterol

Change in age-standardized mean HDL cholesterol

Extended Data Fig. 4 |Change in age-standardized mean HDL and non-HDL cholesterol between 1980 and 2018 by region for women and men. One mmol ${ }^{-1}$ is equivalent to $38.61 \mathrm{mg} \mathrm{dl}^{-1}$. 
Article
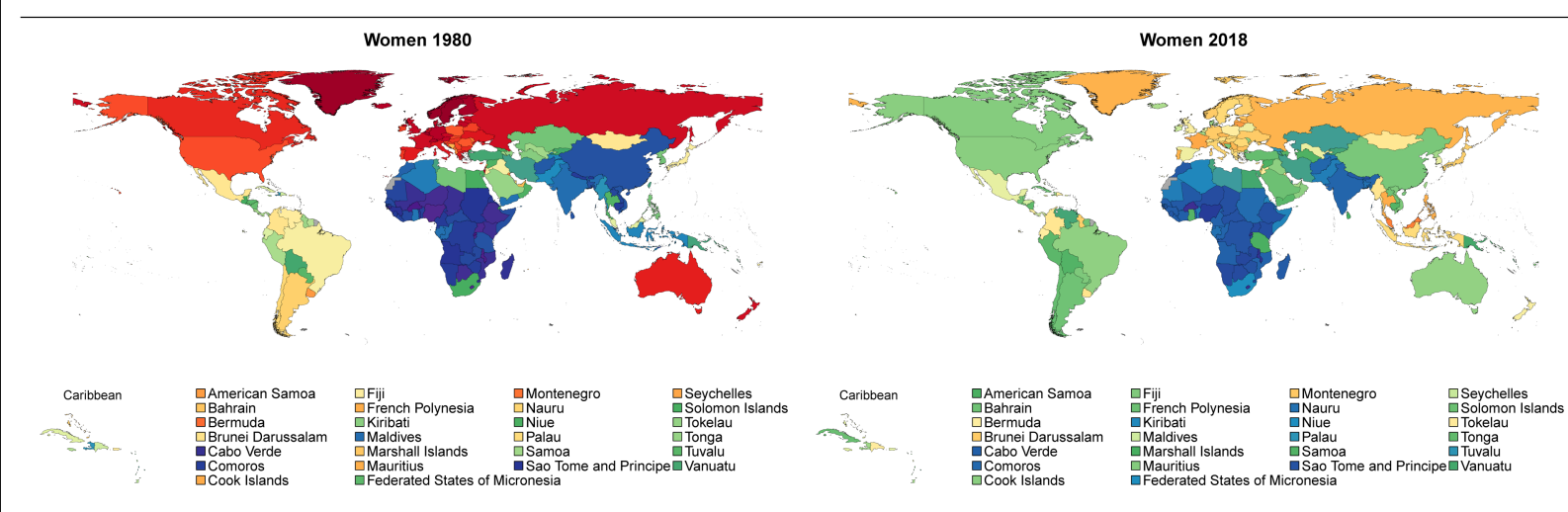

Men 1980
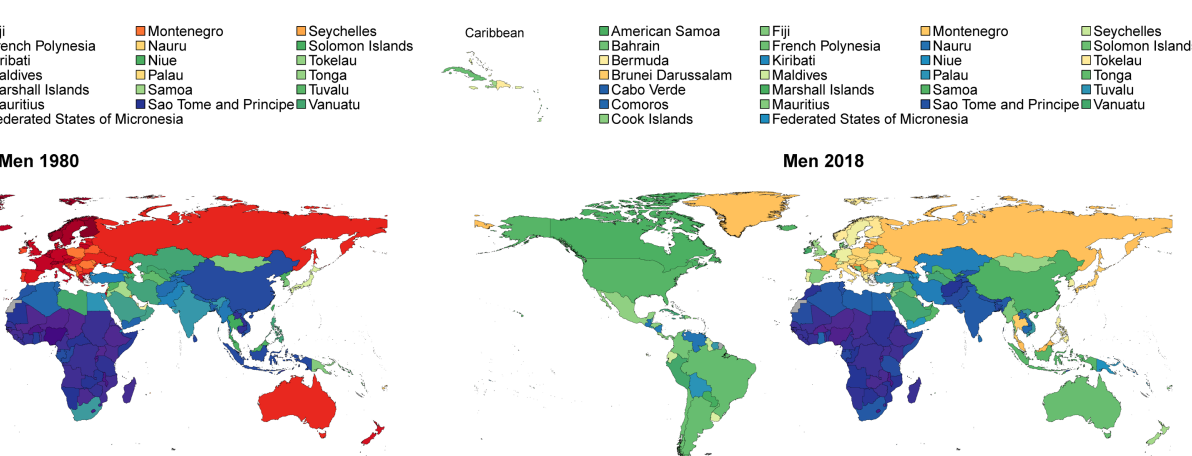

Men 2018<smiles>[B]=C</smiles>

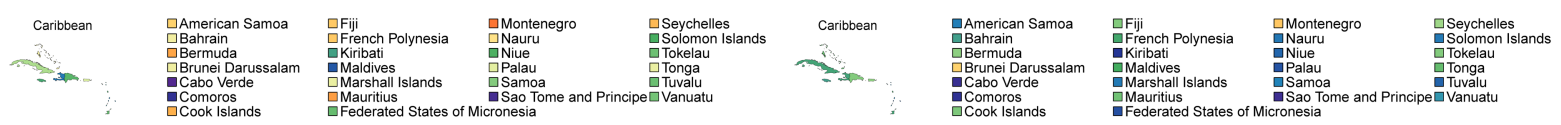

Extended Data Fig. 5 | Age-standardized mean total cholesterol by country in 1980 and 2018 for women and men. One mol ${ }^{-1}$ is equivalent to $38.61 \mathrm{mg} \mathrm{dl}^{-1}$. 

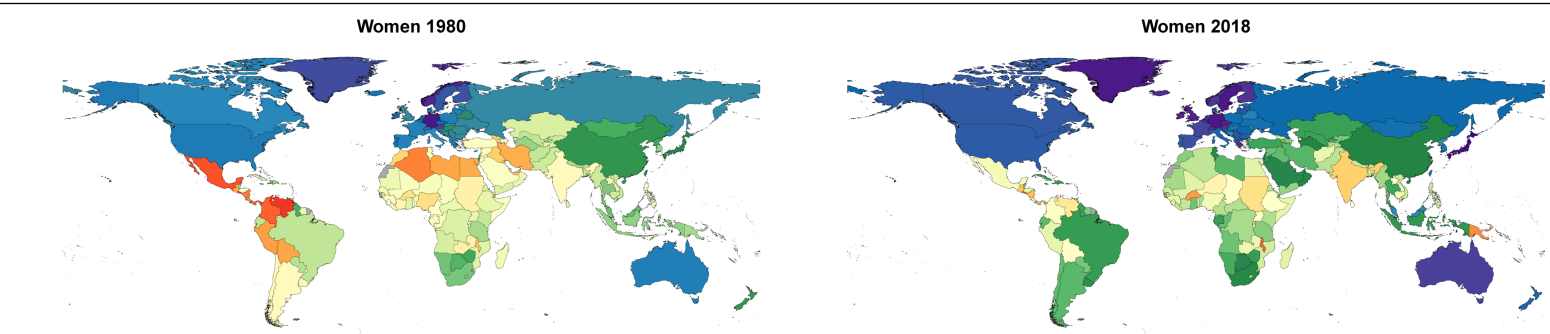

Age-standardized
mean HDL cholestero

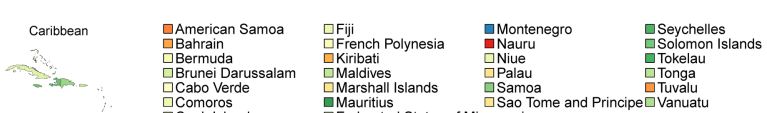

पComoros
$\square$ Cook Islands 口Samo aTuvalu Men 1980
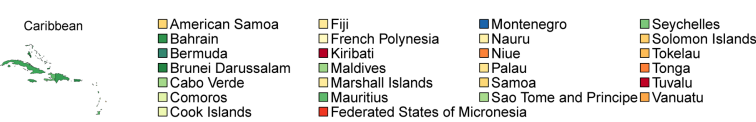
ISamoa
Sao Tome and Principe CVavalu

\section{Men 1980}
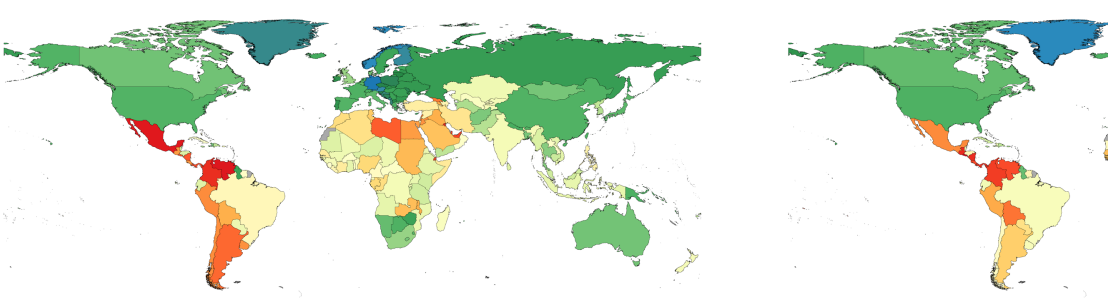

Men 2018

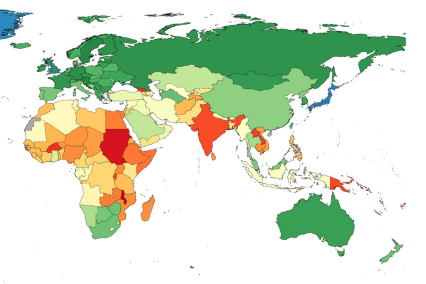

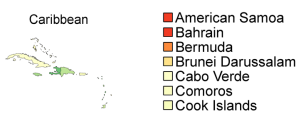
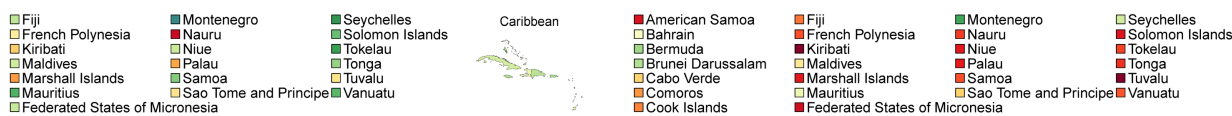

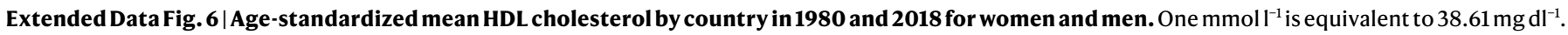




\section{Article}
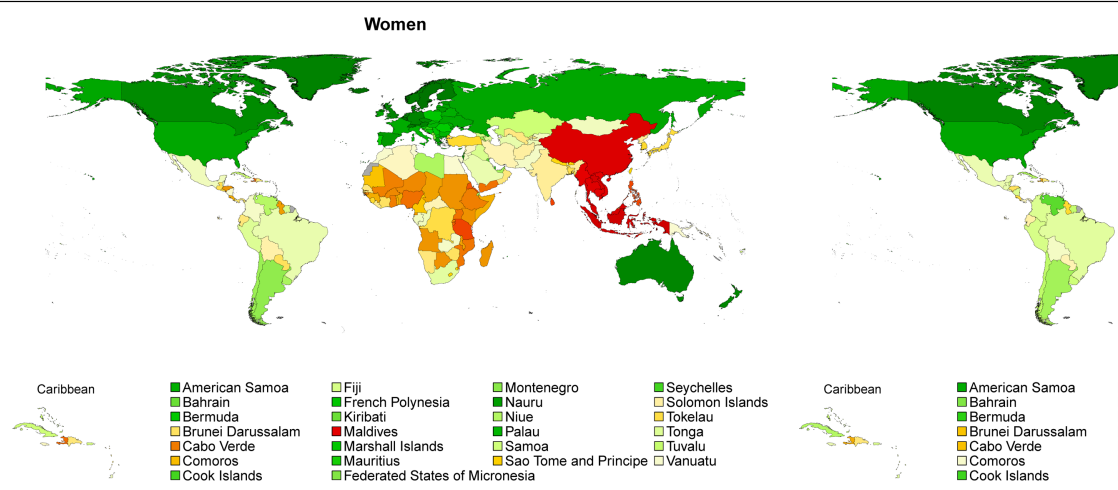

Men

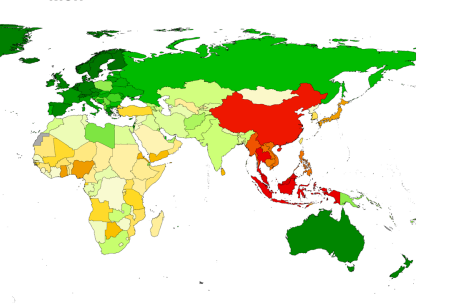

Change in age-standardized mean total cholesterol
(mmol $\mathrm{l}^{-1}$ per decade)
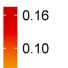

0.00

$-0.10$

$-0.20$
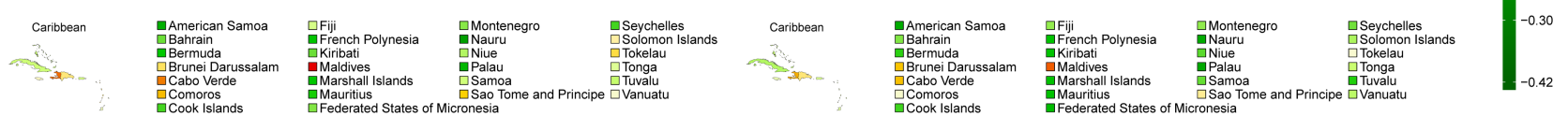

Extended Data Fig. $7 \mid$ Change per decade in age-standardized mean total cholesterol by country for women and men. One $\mathrm{mmol}^{-1}$ is equivalent to $38.61 \mathrm{mg} \mathrm{dl}^{-1}$. 
Women

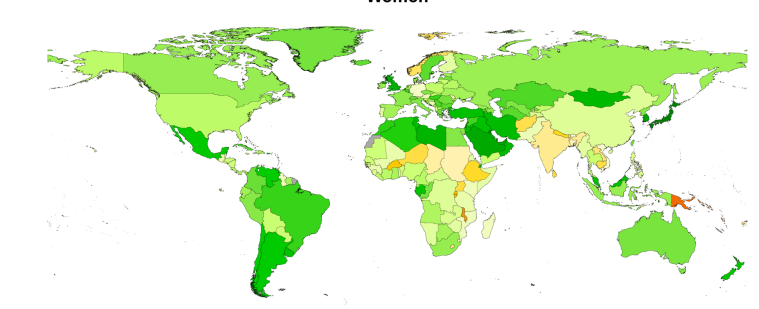

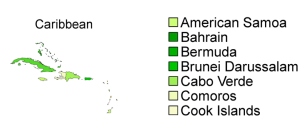

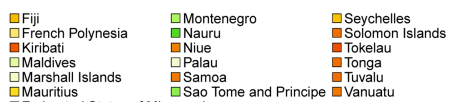

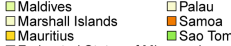
$\square$ Mauritius
口Federated States of Micronesia
Men
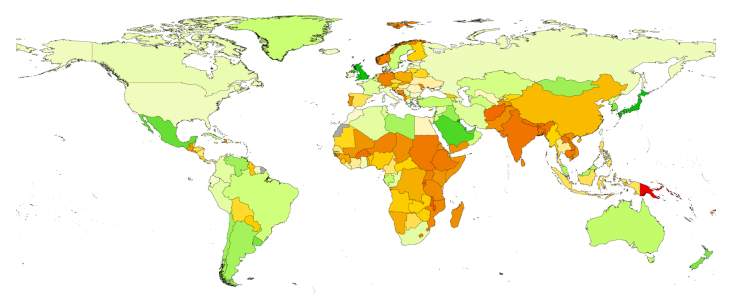

mean $\mathrm{HDL}$ age-standardize

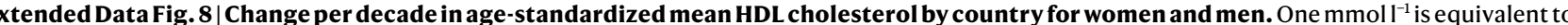
$38.61 \mathrm{mg} \mathrm{dl}^{-1}$. 


\section{Article}

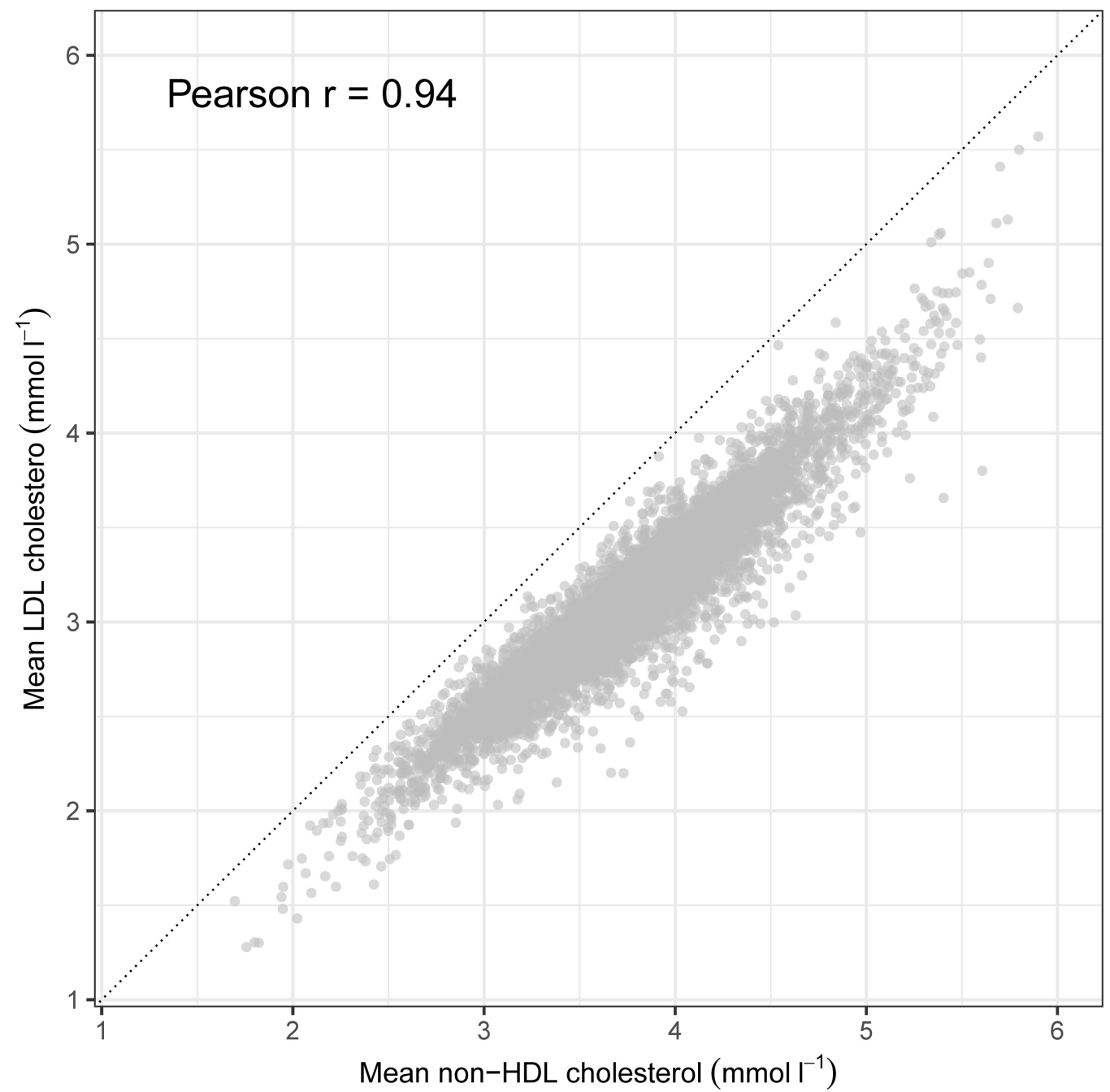

Extended Data Fig. 9 | The association between mean LDL and non-HDL cholesterol in studies that measured lipids in a laboratory that had data for both variables. Each data point is one study-age-sex group $(n=6,864)$. One $\mathrm{mmoll}^{-1}$ is equivalent to $38.61 \mathrm{mg} \mathrm{dl}^{-1}$. 


\section{natureresearch}

Corresponding author(s): Majid Ezzati

Last updated by author(s): Mar 19, 2020

\section{Reporting Summary}

Nature Research wishes to improve the reproducibility of the work that we publish. This form provides structure for consistency and transparency in reporting. For further information on Nature Research policies, see Authors \& Referees and the Editorial Policy Checklist.

\section{Statistics}

For all statistical analyses, confirm that the following items are present in the figure legend, table legend, main text, or Methods section.

n/a Confirmed

Х $\square$ The exact sample size $(n)$ for each experimental group/condition, given as a discrete number and unit of measurement

Х $\square$ A statement on whether measurements were taken from distinct samples or whether the same sample was measured repeatedly

$\square$ The statistical test(s) used AND whether they are one- or two-sided

Only common tests should be described solely by name; describe more complex techniques in the Methods section.

$\searrow \square$ A description of all covariates tested

\ $\square$ A description of any assumptions or corrections, such as tests of normality and adjustment for multiple comparisons

A full description of the statistical parameters including central tendency (e.g. means) or other basic estimates (e.g. regression coefficient)

$\triangle$ AND variation (e.g. standard deviation) or associated estimates of uncertainty (e.g. confidence intervals)

$X$ For null hypothesis testing, the test statistic (e.g. $F, t, r$ ) with confidence intervals, effect sizes, degrees of freedom and $P$ value noted

Give $P$ values as exact values whenever suitable.

$\bigotimes$ For Bayesian analysis, information on the choice of priors and Markov chain Monte Carlo settings

For hierarchical and complex designs, identification of the appropriate level for tests and full reporting of outcomes

Estimates of effect sizes (e.g. Cohen's $d$, Pearson's $r$ ), indicating how they were calculated

Our web collection on statistics for biologists contains articles on many of the points above.

\section{Software and code}

\section{Policy information about availability of computer code}

Data collection $\quad$ Processing of secondary data was conducted using the statistical software R (version 3.6.0).

Data analysis All analyses were conducting using the statistical software R (version 3.6.0). The code for estimation of mean risk factor trends is available at www.ncdrisc.org.

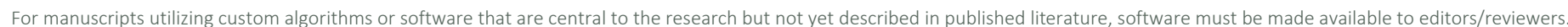
We strongly encourage code deposition in a community repository (e.g. GitHub). See the Nature Research guidelines for submitting code \& software for further information.

\section{Data}

Policy information about availability of data

All manuscripts must include a data availability statement. This statement should provide the following information, where applicable:

- Accession codes, unique identifiers, or web links for publicly available datasets

- A list of figures that have associated raw data

- A description of any restrictions on data availability

This is a data-pooling study that brings together more than 1000 disparate data sources and uses a Bayesian hierarchical model to estimate population risk factor trends. Estimates of mean total, non-HDL and HDL cholesterol by country, year, and sex will be available from www.ncdrisc.org upon the publication of the paper. Some of the input data sources are publicly available, for which we will add links in the final version of the paper. Others are the property of specific research groups and agencies, for which we will provide contact information. 
Please select the one below that is the best fit for your research. If you are not sure, read the appropriate sections before making your selection.
Life sciences
\Behavioural \& social sciences
Ecological, evolutionary \& environmental sciences

For a reference copy of the document with all sections, see nature.com/documents/nr-reporting-summary-flat.pdf

\section{Behavioural \& social sciences study design}

All studies must disclose on these points even when the disclosure is negative.

Study description We pooled and re-analysed population-based data that had measured blood lipids in adults to estimate trends in mean total, non-HDL and HDL cholesterol from 1980 to 2018 for 200 countries and territories, using a Bayesian hierarchical model.

Research sample We pooled data from 1,127 population-based studies of blood lipids conducted in 161 countries, with measurement of blood lipids in over 102 million adults aged 18 years and older. Studies were representative of a national, subnational or community population.

Sampling strategy We included data collected using a probabilistic sampling method with a defined sampling frame. We therefore included studies with simple random and complex survey designs but excluded convenience samples.

Data collection

We used data on measured blood lipids to calculate mean total, non-HDL and HDL cholesterol. We excluded self-reported data.

Timing

Data exclusions

We pooled data collected from 1980 to 2018 . We also included national studies for the 3 years prior to 1980 ( $n=1$ ), assigning them to 1980 , so that they can inform the estimates in countries with slightly earlier national data.

We excluded all data sources that included only hypercholesterolemia or dyslipidaemia diagnosis history or medication status without measurement of cholesterol levels. We also excluded data sources on population subgroups whose lipid profile may differ systematically from the general population, including:

- studies that had included or excluded people based on their health status or cardiovascular risk;

- studies whose participants were only ethnic minorities;

- specific educational, occupational, or socioeconomic subgroups, with the exception noted below;

- those recruited through health facilities, with the exception noted below.

We used school-based data in countries, and in age-sex groups, where secondary school enrollment was $70 \%$ or higher. We used data whose sampling frame was health insurance schemes in countries where at least $80 \%$ of the population were insured. Finally, we used data collected through general practice and primary care systems in high-income and central European countries with universal insurance, because contact with the primary care systems tends to be as good as or better than response rates for population-based surveys. Our exclusion criteria were established at the initiation of the study to ensure all data were representative.

Non-participation Our inclusion/exclusion criteria were designed to ensure participants of the surveys included were representative of the general population from which each sample was drawn.

Randomization

Our study is descriptive, and we did not carry out experiments.

\section{Reporting for specific materials, systems and methods}

We require information from authors about some types of materials, experimental systems and methods used in many studies. Here, indicate whether each material, system or method listed is relevant to your study. If you are not sure if a list item applies to your research, read the appropriate section before selecting a response.

\begin{tabular}{|c|c|}
\hline $\mathrm{n} / \mathrm{a}$ & Involved in the study \\
\hline Х & $\square$ Antibodies \\
\hline$\bigotimes$ & $\square$ Eukaryotic cell lines \\
\hline$\bigotimes$ & $\square$ Palaeontology \\
\hline$\bigotimes$ & $\square$ Animals and other organisms \\
\hline 邓 & $\square$ Human research participants \\
\hline Х & $\square$ Clinical data \\
\hline
\end{tabular}

\begin{tabular}{l|l} 
Methods \\
\hline n/a & Involved in the study \\
$\searrow$ & $\square$ ChIP-seq \\
$X$ & $\square$ Flow cytometry \\
$\square$ & $\square$ MRI-based neuroimaging
\end{tabular}

